NBER WORKING PAPER SERIES

\title{
THE TIME SERIES OF THE CROSS SECTION OF ASSET PRICES
}

\author{
Lior Menzly \\ Tano Santos \\ Pietro Veronsesi \\ Working Paper 9217 \\ http://www.nber.org/papers/w9217 \\ NATIONAL BUREAU OF ECONOMIC RESEARCH \\ 1050 Massachusetts Avenue \\ Cambridge, MA 02138 \\ September 2002
}

We thank seminar participants at University of Texas at Austin, NYU, University of Illinois at UrbanaChampaign, McGill, MIT, and the University of Chicago. We also thank John Cochrane, George Constantinides, Lars Hansen, John Heaton, Martin Lettau and Jessica Wachter for their comments. The views expressed herein are those of the authors and not necessarily those of the National Bureau of Economic Research.

(C) 2002 by Lior Menzly, Tano Santos, and Pietro Veronesi. All rights reserved. Short sections of text, not to exceed two paragraphs, may be quoted without explicit permission provided that full credit, including (C) notice, is given to the source. 
The Time Series of the Cross Section of Asset Prices

Lior Menzly, Tano Santos, and Pietro Veronesi

NBER Working Paper No. 9217

September 2002

JEL No. G1

\begin{abstract}
In this paper we propose a general equilibrium model that successfully reproduces the historical experience of the cross section of US stock prices as well as the realized history of the market portfolio. The model achieves this while addressing traditional concerns in the asset pricing literature: A high equity premium and volatility of returns, the long horizon predictability, and a low volatility of the risk free rate. The model combines a rich payoff structure with a habit persistence discount factor, which allows us to identify the effect on prices of idiosyncratic cash flow shocks versus business cycle components.
\end{abstract}

Lior Menzly

University of Chicago

1101 East $58^{\text {th }}$ Street

Chicago, IL 60637

Pietro Veronesi

Graduate School of Business

University of Chicago

1101 East $58^{\text {th }}$ Street

Chicago, IL 60637

and NBER, CEPR

pietro.veronesi@gsb.uchicago.edu
Tano Santos

Graduate School of Business

University of Chicago

1101 East $58^{\text {th }}$ Street

Chicago, IL 60637

and NBER

jesus.santos@gsb.uchicago.edu 


\section{INTRODUCTION}

What are the sources of differences across asset prices? How does the cross section of stock prices behave over the business cycle? Do prices show secular trends and, if so, what is the source of this trend? Asset prices are the result of the interaction of cash flows with the stochastic discount factor and, consequently, answers to these questions rest on a thorough understanding of each of these two components. Surprisingly though, there has been little theoretical work combining a rich payoff structure with an interesting pricing kernel in a general equilibrium framework, and, hence, these issues have not been successfully addressed in the literature. The purpose of this paper is to fill this void. Specifically, we write a general equilibrium model that tightly links the cross section and the time series of asset prices to cash flows and a consumption based stochastic discount factor. The model successfully reproduces the historical experience of the cross section of US stock prices as well as the realized history of the market portfolio. In addition, our set up is able to address traditional concerns in the asset pricing literature: A high equity premium and volatility of returns, the long horizon predictability, and a low volatility of the risk free rate.

To gain some insight on the interaction between cash flows and stochastic discount factors, Figure 1 shows evidence on the relation between the cross section of asset prices and the business cycle. Panel A shows the log of the price dividend ratio of the market portfolio versus the cross sectional standard deviation of the log price dividend ratios of industry-sorted portfolios for the sample period 1927-2001. Both series show a great deal of comovement and indeed their correlation is a solid .75. As it is well known variation in the price dividend ratio of the market portfolio is mostly driven by shocks to expected rates of return, an aggregate effect. The high correlation between these two series then suggests a link between the aggregate factors driving the time series variation of the market portfolio and the cross sectional dispersion across industries. Moreover, Panel B, where we plot the same series in first differences, shows that the same pattern holds for higher frequencies. Once again both series show a great deal of comovement and their correlation coefficient is still a healthy .40.

Clearly, price dividend ratio fluctuations can only be driven by news to cash flows and news to discount rates, so whatever variation there is in the cross section of these ratios must be driven by differences on how each industry loads in either one of these two components. We can then apply the traditional tools of variance decomposition literature to deepen our 
understanding of the driving forces behind the time variation in the cross sectional dispersion. Table I Panel A reports the percentage of the variance of log price dividend ratio that is driven by shocks to expected cash flows versus shocks to expected returns for two industries in our sample, Railroads and Paper. For comparison we include the more standard variance decomposition for the market portfolio. ${ }^{1}$ Paper and Railroads differ markedly. For example, while Paper is similar to the market portfolio, as shocks to discount rates make up the largest component of the variance of its log price-dividend ratio, this is not true for Railroads, where the cash flow component seems to have a bigger role (although statistically insignificant). A similar exercise across industries reveals that for some industries the variance of log pricedividend ratios is driven to a large extent by cash flow news whereas for others they explain a much smaller fraction of this variance.

Table I Panel B reports the same result from the perspective of standard five year ahead predictability regressions. Price dividend ratios predict future returns in the case of Paper, very much like (or even better than) the well known predictability result for the market portfolio, whereas they do not in the case of Railroads.

The variance decomposition and predictability regressions are ideal instruments to analyze what drives the price dividend ratios of various industries and summarize their cross sectional differences conveniently, but we are interested in another aspect of the data as well, namely, the level and trend of asset prices. To illustrate this Figure 2 plots the price of Railroads and Paper, normalized both by the level of US consumption. For comparison, we have included the price consumption ratio of the market portfolio. The pattern is striking: while Railroads has experienced a severe secular decline, Paper experienced a surge in the mid fifties and has remained roughly steady ever since. These two industries differ only in their cash flow realizations and we are interested in finding out how far can these cash flows go in rationalizing the observed histories.

Our model will be able to shed light on the cross-sectional differences in predictability

\footnotetext{
${ }^{1}$ The voluminous literature on the variance decomposition starts with Campbell and Shiller's (1988a,b) seminal contribution. See also Cochrane (1991). The general finding is that the cash flow component does not explain much of the variation of the log price-dividend ratio of the market portfolio, while the return component explains a larger percentage of it. Vuoltenahoo (2002) conducts the same exercise at the firm level, finding that, in contrast to the market portfolio, individual stock returns are mainly driven by news to cash flows. Ours is the same exercise at an intermediate level of aggregation.
} 
and variance decomposition, and, in the process, account for the diverse historical experiences of the industries in our data set. Specifically, we write an economy with a representative agent whose preferences are the log version of the habit persistence model of Campbell and Cochrane (1999). As these authors have shown, these preferences induce fluctuations in the stochastic discount factor that can successfully explain the main patterns of aggregate market data. ${ }^{2}$

The novel aspect of this paper is the model of cash flow we introduce, a necessary ingredient if prices are the object of interest. ${ }^{3}$ In our set up the representative consumer can trade multiple dividend paying assets. Rather than modeling the level of dividends themselves, we specify processes for the fraction that each asset contributes to total consumption. These processes are stationary and bounded between zero and one. This modeling device then naturally induces dividend series that add to a consumption process that is consistent with the observed behavior of the US time series. ${ }^{4}$ Moreover the framework, while rich in implications, is tractable enough to yield pricing formulas that are interpretable and that are directly linked to fundamentals.

In particular, we find that asset prices are given by a linear function of a stochastic trend in dividends plus a second term that reflects deviations from this trend. The loadings on the stochastic trend and the actual dividends vary with the business cycle, as they increase

\footnotetext{
${ }^{2}$ The literature on habit persistence and asset pricing is large and includes, in addition to Campbell and Cochrane (1999), Sundaresan (1989), Constantinides (1990), Abel (1990), Ferson and Constantinides (1991), Detemple and Zapatero (1991), Daniel and Marshall (1997), and Li (2001). The habit persistence literature though has largely concentrated on the time series properties of the market portfolio rather than on the cross section, the main focus of the present work. Wachter (2000 and 2001) is an exception as she studies the bond pricing implications of the habit persistence model of Campbell and Cochrane (1999), effectively pricing bonds and the the aggregate market portfolio.

${ }^{3}$ As mentioned modeling cash flows has elicited very little research. An early reference in this direction is Bossaerts and Green (1990) who model dividend processes directly. Abel (1999) and Bansal, Dittmar, and Lundblad (2001) have proposed a consumption leverage model where an asset's premium is determined by the extent to which its dividend loads on consumption. None of these papers though attempt to address the time series variation of the cross section of stock prices'. Furthermore, these models are not fully fledged general equilibrium model nor are the cash flow processes defined in such a way as to prevent assets to become irrelevant as a fraction of total consumption or to account for more than it. Our model is more closely related to the general equilibrium set up of Brock (1982), though his is a production economy framework.

${ }^{4}$ The model is rich enough to accommodate sources of income other than financial. Indeed Santos and Veronesi (2001) use the technology introduced in this paper to shed light on the connection between labor income and asset prices.
} 
during peaks and decrease during troughs. Cash flow shocks then have a very different effect on an asset's price depending on where the economy is over the business cycle. Conversely, aggregate shocks affect assets differently depending on whether their current dividends are above or below the stochastic trend. In addition, prices depend on the covariance between the share of consumption that the asset commands and consumption growth and once again the weight of this component depends on where the economy is over the business cycle inducing an additional source of conditional and unconditional cross sectional variation. We are able then to offer a complete characterization of the cross section of asset prices as a function of both aggregate and idiosyncratic cash flow shocks.

In this paper we are interested in the connection between fundamentals and the level of prices, a distinct information not captured in returns. This can only be done if one specifies a cash flow model. Because this is the novel contribution of the paper we simply sketch the implications of our set up for returns. A thorough theoretical and empirical analysis of these implications can be found in a companion paper. (Menzly, Santos, and Veronesi (2002)).

In order to asses the empirical validity of our setup we need to obtain values for the different parameters of the model. As in Campbell and Cochrane (1999), we calibrate the preference parameters to match basic moments of the aggregate market portfolio and the interest rate. In addition, we use dividend and consumption data from 1927-2001 to estimate the parameters of the cash-flow model for the industry-sorted portfolios. Importantly, we avoid the use of any information contained in individual prices or returns to obtain these parameters, otherwise the assessment of the model's ability to account for the main empirical properties of the cross section would be compromised.

We then conduct two sets of experiments. First, we run simulations of our economy. This will allow us to compute moments for which we have no closed form solutions. In particular, given that our set up explicitly models cash flows and discount factors, we want to asses whether our model can account for the predictability of returns across industries as well as the variance decomposition of their price dividend ratios, as in Table I and Figures 1 and 2. Our model provides a striking account of these cross sectional differences.

Second, we investigate whether the model can reproduce the observed US financial history. To do so we feed the pricing formulas found in the theoretical section the realized consumption and cash flow shocks. We show that the model offers a remarkable account of 
the observed history of the prices of industry portfolios, an account, recall, that is simultaneously consistent with the behavior of the aggregate market. Furthermore, we show as well that the model naturally generates the positive correlation between the log price dividend ratio of the market portfolio and the time series of the cross sectional standard deviation of log price dividend ratios. The model performs well even when both times series are in first differences, accounting for the historical patterns in Figure 1, Panels A and B.

The paper proceeds as follows. Section II covers the model, which is solved in Sections III and IV. The model's calibration is reported in Section V. Section VI evaluates the model. Section VII ties some loose ends in the model and Section VIII concludes. All proofs are contained in the Appendix.

\section{THE MODEL}

\section{II.A Preferences}

The economy is composed of a representative consumer who maximizes:

$$
\mathbf{E}\left[\int_{0}^{\infty} u\left(C_{t}, X_{t}, t\right) d t\right]=\mathbf{E}\left[\int_{0}^{\infty} e^{-\phi t} \log \left(C_{t}-X_{t}\right) d t\right],
$$

where $X_{t}$ denotes the habit level and $\phi$ denotes the subjective discount rate. ${ }^{5}$

The effect of habit persistence on the agent's attitudes towards risk can be conveniently summarized by the surplus consumption ratio, $S_{t}$, defined as:

$$
S_{t}=\frac{C_{t}-X_{t}}{C_{t}}
$$

Movements of this surplus directly translate into fluctuations of the local curvature of the utility function, $1 / S_{t}$. As can readily be checked, a low $S_{t}$ is associated with "bad states," periods of high risk aversion, whereas a high $S_{t}$ corresponds to "good states," periods of low

\footnotetext{
${ }^{5}$ The idea of habit formation dates back to Duesenberry (1949). Deaton and Muellbauer (1980) provide a survey and early references. These preferences have been used in a rich variety of contexts. Some applications include Boldrin, Christiano, and Fisher (2001), Fuhrer (2000), or Lettau and Uhlig (2000) in the RBC literature, Bakshi and Chen (1996) and Carroll, Overland, and Weil (2000) in the growth literature. The asset pricing applications are many and were briefly cited the previous footnote. These preferences have generated a remarkable amount of empirical work, for instance, Muellbauer (1988), Heien and Durham (1991), Heaton (1993), and Dynan (2000).
} 
risk aversion. These fluctuations naturally translate into the corresponding variation on the prices and returns of financial assets.

\section{II.B The cash flow model}

There are $n$ risky assets, not all necessarily financial, paying a dividend rate $\left\{D_{t}^{i}\right\}_{i=1}^{n}$, in units of a homogeneous and perishable consumption good. These dividends must add up to a process that is consistent with the observed time series of US consumption. For this reason, and in accordance with the empirical evidence, we assume that the process for aggregate consumption growth can be well approximated as an i.i.d. process:

$$
\frac{d C_{t}}{C_{t}}=\mu_{c} d t+\sigma_{c} d \mathbf{B}_{t}
$$

where $\mu_{c}$ is the mean consumption growth, $\sigma_{c}$ is a $1 \times n$ dimensional vector, and $\mathbf{B}_{t}$ denotes a $n \times 1$ dimensional standard Brownian Motion vector. Furthermore, without loss of generality we impose that

$$
\sigma_{c}=\left(\sigma_{c, 1}, 0, \cdots, 0\right)
$$

Instead of positing processes for $\left\{D_{t}^{i}\right\}_{i=1}^{n}$ that add up to the aggregate consumption process (1), we model the shares of consumption that are generated by each of asset

$$
s_{t}^{i}=\frac{D_{t}^{i}}{C_{t}}
$$

as a mean reverting process of the form

$$
d s_{t}^{i}=\kappa\left(\bar{s}^{i}-s_{t}^{i}\right) d t+s_{t}^{i}\left(\mathbf{v}^{i}-\sum_{j=1}^{n} s_{t}^{j} \mathbf{v}^{j}\right) d \mathbf{B}_{t},
$$

for $i=1, \cdots, n$, where $\bar{s}^{i}$ is the average share of the endowment source $i, \kappa$ is the speed of mean reversion and $\mathbf{v}^{i}$ for $i=1, \cdots, n$ are $n$ dimensional vectors of constants. The form of the volatility function is chosen in order to guarantee that $s_{t}^{i}>0$ for $i=1, \cdots, n$ and $\sum_{i=1}^{n} s_{t}^{i}=1$ for all $t>0 .^{6}$

We notice two important features of the diffusion part in process (4): $(i)$ It is parametrically indeterminate, that is, adding a constant vector, $\mathbf{c}$, to all the $\mathbf{v}^{i}$ 's leaves the share

\footnotetext{
${ }^{6}$ The assumption that the mean reversion speed, $\kappa$, is common across shares is made to simplify the presentation and can be easily generalized. The restriction of a common mean reversion speed though cannot be rejected in the data set we employ in this paper.
} 
processes unaltered; (ii) Since $s_{t}^{n}=1-\sum_{j=1}^{n-1} s_{t}^{j}$, one only needs to specify $n-1$ vectors $\mathbf{v}^{i}$, $i=1, . ., n-1$ to completely describe the share process. In other words, we can assume $\mathbf{v}^{n}=0$ without loss of generality.

In what follows, we choose to renormalize the $\mathbf{v}^{i}$ s, for $i=1, \ldots, n$ in order to minimize the effect of the term $\sum_{j=1}^{n} s_{t}^{j} \mathbf{v}^{j}$ in the diffusion part of (4). We can accomplish this by simply setting $\mathbf{c}$ in $(i)$ such that unconditionally

$$
\sum_{j=1}^{n} \bar{s}^{j} \mathbf{v}^{j}=0
$$

\section{II.C Habit dynamics}

The particular assumptions on the dynamics of $S_{t}$ are of critical importance in habit persistence models. In particular, Campbell and Cochrane (1999) assume that $\log \left(S_{t}\right)$ follows a mean reverting process with shocks that are conditionally perfectly correlated with consumption growth. We depart from Campbell and Cochrane (1999) and impose the stochastic structure instead on the inverse of the surplus consumption ratio, which we denote $Y_{t}$,

$$
Y_{t}=\frac{1}{S_{t}}=\frac{C_{t}}{C_{t}-X_{t}}=\frac{1}{1-\left(\frac{X_{t}}{C_{t}}\right)}>1 .
$$

Throughout we refer to $Y_{t}$ as the inverse surplus. We assume that it follows a mean reverting process:

$$
d Y_{t}=k\left(\bar{Y}-Y_{t}\right) d t-\alpha\left(Y_{t}-\lambda\right)\left(\frac{d C_{t}}{C_{t}}-\mu_{c} d t\right),
$$

where $\bar{Y}$ is the long run mean of the inverse surplus and $k$ is the speed of the mean reversion: ${ }^{7}$

$$
\mathbf{E}_{t}\left[Y_{\tau}\right]=\bar{Y}+\left(Y_{t}-\bar{Y}\right) e^{-k(\tau-t)}
$$

The parameter $\alpha>0$ captures the impact of consumption growth shocks on the inverse surplus process. A negative shock to consumption growth, for example, results in an increase in the inverse surplus, or, equivalently, a decrease in the surplus level. Finally, the parameter $\lambda \geq 1$ ensures a lower bound for the inverse surplus, and an upper bound for the surplus itself. For instance, if the surplus $S_{t}$ is to live in $[0, .1]$ (as in the calibration of Campbell and Cochrane (1999)) then $Y_{t} \in[10, \infty)$, and this can be guaranteed by setting $\lambda=10$. Furthermore, this modeling device avoids the concerns raised by Chapman (1998), namely, the possibility of negative marginal utility of consumption in habit persistence endowment economies.

\footnotetext{
${ }^{7}$ The stationary density of the inverse surplus process can be found in the Appendix.
} 


\section{ASSET PRICES}

We assume that markets are complete and, as a consequence, the standard asset pricing formula holds. That is, if $g_{\tau}$ is the payment of a particular asset at time $\tau$, then the price at time $t$ is:

$$
\begin{aligned}
P_{t}^{g} & =\mathbf{E}_{t}\left[\int_{t}^{\infty} e^{-\phi(\tau-t)}\left(\frac{u_{c}\left(C_{\tau}-X_{\tau}\right)}{u_{c}\left(C_{t}-X_{t}\right)}\right) g_{\tau} d \tau\right] \\
& =\left(C_{t}-X_{t}\right) \mathbf{E}_{t}\left[\int_{t}^{\infty} e^{-\phi(\tau-t)}\left(\frac{g_{\tau}}{C_{\tau}-X_{\tau}}\right) d \tau\right] .
\end{aligned}
$$

Let $s_{\tau}^{g}=g_{\tau} / C_{\tau}$ the share of consumption of the asset that represents a claim to $g_{\tau}$. Then straightforward manipulations of equation (9) yield:

$$
\frac{P_{t}^{g}}{C_{t}}=\frac{1}{Y_{t}} \mathbf{E}_{t}\left[\int_{t}^{\infty} e^{-\phi(\tau-t)} s_{\tau}^{g} Y_{\tau} d \tau\right]
$$

Calculation of the prices then requires computation of the expectation in expression (10) for the assets of interest. Rather than show prices as a function of $Y_{t}$, as in (10), we present the results in terms of the surplus consumption ratio, $S_{t}$, for this is the convention adopted by Campbell and Cochrane (1999) and others after them (see Li (2001) and Wachter (2000)).

\section{III.A. The price of the total wealth portfolio}

The total wealth portfolio gives claim to the whole consumption stream, $g_{\tau}=C_{\tau}$, and hence $s_{\tau}^{g}=1$. In this case, the application of equation (8) in the pricing equation (10) leads immediately to the following proposition: ${ }^{8}$

Proposition 1. The price consumption ratio of the total wealth portfolio is given by:

$$
\frac{P_{t}^{T W}}{C_{t}}=\frac{1}{\phi+k}\left[1+\frac{k \bar{Y}}{\phi} S_{t}\right] .
$$

Equation (11) neatly captures the mechanism embedded in habit persistence models. A positive consumption shock increases $S_{t}$, as it is perfectly correlated with consumption growth, and this results in an increase of the price consumption ratio. The reason is that a positive shock in consumption growth makes the investor less risk averse and hence the price of the total wealth portfolio is bid up relative to the pre-shock level.

\footnotetext{
${ }^{8}$ Throughout the paper, we assume that the parameters for the process for the inverse surplus $Y_{t}$ satisfy the integrability condition $\mathbf{E}_{t}\left[\int_{t}^{\infty} e^{-\phi(\tau-t)} Y_{\tau} d \tau\right]<\infty$. This is sufficient to invoke Fubini Theorem to justify the inversion of integration and expectation implicit in the derivation of (11).
} 
Clearly, if $\alpha=0$ and $Y_{t}=\bar{Y}=\lambda=1$, then $S_{t}=1 / \bar{Y}$ for $t \geq 0$ and the price consumption ratio of the total wealth portfolio is given by $1 / \phi$, the standard price consumption ratio of the log economy (Rubinstein (1976)). ${ }^{9}$

\section{III.B The price of individual securities}

Now $g_{\tau}=D_{\tau}^{i}$ and $s_{\tau}^{g}=s_{\tau}^{i}$, the share of asset $i$. Since in the case of individual securities the interaction between cash-flow effects and discount effects is difficult to disentangle, we solve for two simpler cases first. The first one, in section III.B.1, shuts off the discount-effect channel, that is, the habit, but it allows for a rich cross-sectional variation in cash-flow betas. The second case, in section III.B.2, assumes equal cash flow betas but it allows for habit effects. Section III.B.3 finally combines the two cases.

\section{III.B.1 Model A: Prices in the absence of habit persistence}

To shut down the effect of the variation in the degree of risk aversion we must have $X_{t}=0$ for all $t$. Hence, we simply set

$$
\text { Model A } \quad \alpha=0 \quad \text { and } \quad Y_{t}=\bar{Y}=\lambda=1
$$

We then obtain the standard log utility representation with multiple assets. The prices of individual securities then follow easily: since in equation (10) we have $s_{\tau}^{g} Y_{\tau}=s_{\tau}^{i}$ and from (4)

$$
\mathbf{E}_{t}\left[s_{\tau}^{i}\right]=\bar{s}^{i}+\left(s_{t}^{i}-\bar{s}^{i}\right) e^{-\kappa(\tau-t)}
$$

by substituting (13) into (10) we find: ${ }^{10}$

Proposition 2. (Prices under Model A.) Let $\alpha=0$ and $Y_{t}=\bar{Y}=\lambda=1$, then the price of asset $i, P_{t}^{A, i}$, is given by

$$
P_{t}^{A, i}=\frac{\bar{D}_{t}^{i}}{\phi}+\frac{D_{t}^{i}-\bar{D}_{t}^{i}}{\phi+\kappa}
$$

where $\bar{D}_{t}^{i}=\bar{s}^{i} C_{t}$.

$\bar{D}_{t}^{i}$ is the level dividends would have given current consumption in the absence of any idiosyncratic shock and it is a stochastic trend to which dividends revert. Prices are then

\footnotetext{
${ }^{9}$ Notice though that the unconditional mean of the price consumption ratio of the total wealth portfolio is not $\frac{1}{\phi}$, as one has to take into account the Jensen inequality term when taking expectations in (11).

${ }^{10}$ Since $0<s_{t}^{i}<1$ for all $t$, we can invoke Fubini Thorem to justify the inversion of expectation and the integration implicit in the derivation of (14).
} 
driven by a common factor, which is the current level of consumption scaled by the average share, and an idiosyncratic factor which is the distance between the current dividend level, $D_{t}^{i}$, and the stochastic trend. The first term accounts for the differences in levels across prices on average for a given level of consumption and it is discounted by $1 / \phi$. The second term is responsible for the cross sectional variation observed in prices and it is discounted by the sum of $\phi$ plus the speed of mean reversion, $\kappa$. Notice that although the assets are characterized by different covariances of their own dividend growth with consumption growth, that is, different $\theta^{i}=\mathbf{v}^{i} \sigma_{c}$, this last parameter does not enter in the pricing function. The reason is that under log-utility, the discount factor effects offset cash flow effects. This parameter, however, affects the time-series of the dividends with respect to consumption growth and hence, as we shall see, the expected excess returns on this stock.

Armed with expression (14) we can immediately compute the price dividend ratio,

$$
\frac{P_{t}^{A, i}}{D_{t}^{i}}=\left(\frac{1}{\phi+\kappa}\right)\left[1+\left(\frac{\kappa}{\phi}\right) \frac{\bar{s}^{i}}{s_{t}^{i}}\right] .
$$

It follows immediately from (15) that predictable changes in the cross section of price dividend ratios over the business cycle are exclusively driven by changes in the dispersion of the shares $s_{t}^{i}$ relative to their long term averages. In the presence of consumption growth, those assets whose shares covary positively with consumption growth will experience, on average, declines in the price dividend ratios, whereas those that covary negatively will see an increase in their price dividend ratios.

\section{III.B.2 Model B: Prices in the absence of cash flow effects}

To asses the impact of the variation in the discount factor on the cross section of stock prices we now shut down the "cash flow effect." We do this by requiring that share growth does not covary with consumption growth, $\operatorname{cov}_{t}\left(\frac{d s_{t}^{i}}{s_{t}^{i}}, \frac{d C_{t}}{C_{t}}\right)=0$, that is,

$$
\theta^{i} \equiv \mathbf{v}^{i} \sigma_{c}^{\prime}=0 \text { for all } i=1, . ., n
$$

This implies that all assets have identical cash flow betas

$$
\text { Model B } \quad \operatorname{cov}_{t}\left(\frac{d D_{t}^{i}}{D_{t}^{i}}, \frac{d C_{t}}{C_{t}}\right)=\sigma_{c} \sigma_{c}^{\prime} .
$$

As in the previous case, the prices of individual securities are easy to obtain. Since from condition (16), the shares $s_{t}^{i}$ and the inverse surplus $Y_{t}$ are independent processes, we have 
$\mathbf{E}_{t}\left[s_{\tau}^{i} Y_{\tau}\right]=\mathbf{E}_{t}\left[s_{\tau}^{i}\right] \mathbf{E}_{t}\left[Y_{\tau}\right]$. We can then use formulas (13) and (8) and insert them in the pricing equation (10) to easily obtain the result in the following proposition:

Proposition 3. (Prices under Model B.) Let $\operatorname{cov}_{t}\left(\frac{d D_{t}^{i}}{D_{t}^{i}}, \frac{d C_{t}}{C_{t}}\right)=\sigma_{c} \sigma_{c}^{\prime}$, then the price of asset $i, P_{t}^{B, i}$, is given by

$$
P_{t}^{B, i}=\left[1+\frac{k \bar{Y}}{\phi} S_{t}\right] \frac{\bar{D}_{t}^{i}}{\phi+k}+\left[1+\left(\frac{k \bar{Y}}{\phi+\kappa}\right) S_{t}\right] \frac{D_{t}^{i}-\bar{D}_{t}^{i}}{\phi+\kappa+k} .
$$

As in expression (14) there are two terms in the price formula. The first one captures the variation due to the common factor. Now the effect of shocks in consumption, which affect $\bar{D}_{t}^{i}=\bar{s}^{i} C_{t}$, is amplified whenever the surplus is relatively high, as investors are in this case less risk averse. The second term captures the source of idiosyncratic variation, both in the time series and the cross section. Once again idiosyncratic shocks to dividends will be amplified whenever $S_{t}$ is high.

Similarly to Model A, straightforward manipulations of equation (18) yield the following expression for the price dividend ratio,

$$
\frac{P_{t}^{B, i}}{D_{t}^{i}}=\frac{1}{\phi+k}\left[1+\left(\frac{k \bar{Y}}{\phi}\right) S_{t}\right]\left(\frac{\bar{s}^{i}}{s_{t}^{i}}\right)+\frac{1}{\phi+\kappa+k}\left[1+\left(\frac{k \bar{Y}}{\phi+\kappa}\right) S_{t}\right]\left(1-\frac{\bar{s}^{i}}{s_{t}^{i}}\right) .
$$

Model B has very different implications for the behavior of the cross section of price dividend ratios over the business cycle when compared to Model A. Recall that in the latter predictable changes in the cross section were exclusively driven by changes in the distribution of shares due to their covariance with consumption growth, the channel that is shut down here. In contrast, now cash flow shocks have a very different effect on the cross section depending on whether they occur during business cycle peaks or troughs. As can be immediately seen from expression (19), cash flow shocks are magnified during peaks and dampened in troughs. Moreover, as the next proposition shows, even in the absence of cash flow shocks the cross section of price dividend ratios is sensitive to the business cycle.

Proposition 4. For a given distribution of shares, the cross sectional variance of price dividend ratios is an increasing function of the surplus consumption ratio.

That is, the cross sectional dispersion of the price dividend ratios increases during business cycle peaks and decreases during troughs. 


\section{III.B.3 Prices in the general case}

The model in the general case is considerably more complex than Models A and B, and a closed form solution is not available. Instead we offer an approximate solution, where the nature of the approximation is sketched in Section VII. Numerical computation of prices show the approximation is within $0.1 \%$ of the true price for the set of portfolios we consider. Prices for the general case then are given by

$$
P_{t}^{i} \approx \omega^{i} P_{t}^{A, i} \lambda S_{t}+\left(1-\omega^{i}\right) P_{t}^{B, i}
$$

where

$$
\omega^{i}=\frac{\alpha \theta_{i}}{\phi+k+\kappa+\alpha \theta_{i}},
$$

and we recall that $\theta^{i}=\mathbf{v}^{i} \sigma_{c}^{\prime}$. Under condition (5), $\theta^{i}$ is simply the unconditional covariance between the growth rate of shares $d s_{t}^{i} / s_{t}^{i}$ and consumption growth $d C_{t} / C_{t} \cdot{ }^{11}$ In equation (21), $\phi+k+\kappa+\alpha \theta_{i}$ has the interpretation of a discount rate and it is assumed to be positive. The sign of $\omega^{i}$ is then solely determined by the sign of $\theta^{i}$.

In order to better interpret equation (20) it is useful to write it as:

$$
P_{t}^{i} \approx \omega^{i} P_{t}^{A, i}+\left(1-\omega^{i}\right) P_{t}^{B, i}+\omega^{i}\left(\lambda S_{t}-1\right) P_{t}^{A, i}
$$

The price of asset $i$ is a linear combination of the price of the asset found for Model A and that of Model B plus a correction that interacts the surplus consumption ratio with the price of the asset under Model A. To interpret further the last term of equation (22) recall first that $S_{t} \lambda \leq 1$. Assume then that asset $i$ 's share covaries positively with consumption growth, $\omega^{i}>0$. In this case the asset provides little insurance against adverse shocks in consumption growth as the asset pays on average little in those states. As a consequence

$$
\omega^{i}\left(\lambda S_{t}-1\right) P_{t}^{A, i}<0
$$

and the asset trades at a discount relatively to an otherwise identical asset with zero or negative covariance with consumption growth. This effect enters multiplicatively the price of the asset under Model A, which allows for a general covariance structure of dividend growth with consumption growth. Roughly, one can interpret the expression $\omega^{i}\left(\lambda S_{t}-1\right)$ as the correction one needs to add to the price of the asset that corresponds to the logarithmic economy when the

\footnotetext{
${ }^{11}$ This follows immediately since $\sum_{i=1}^{n} \bar{s}^{i} \mathbf{v}^{i} \sigma_{c}^{\prime}=\sigma_{c, 1} \sum_{i=1}^{n} \bar{s}^{i} v_{i, 1}=0$, where we have used expression (2).
} 
degree of risk aversion is different than one. Moreover the magnitude of the insurance discount depends on where the economy finds itself over the business cycle. In troughs, $\left|\lambda S_{t}-1\right|$ is very large and so is the discount of the asset whose cash flows covary positively with consumption growth. When the economy experiences positive consumption growth shocks $S_{t}$ increases and the price discount $\left|\lambda S_{t}-1\right|$ diminishes accordingly.

From equation (20), it is immediate to obtain an expression for the price-dividend ratio in the general case. Since the formula can be readily computed from (15) and (19), we avoid repeating it here. Importantly, though, in the general case we can still expect that a result similar to Proposition 4 holds. However, the contemporaneous correlation of shares $s_{t}^{i}$ and surplus $S_{t}$ makes it hard to obtain a general proposition. A thorough assessment of the model on this dimension is then postponed until Section VII.

\section{ASSET RETURNS}

In this section we compute the risk free rate and the expected excess rate of return of the total wealth portfolio and individual securities. We solve for these quantities insofar as they permit a better understanding of the relationship between cashflows, discount factors, and asset prices, as explained in the introduction. The reader is referred to Menzly, Santos, and Veronesi (2002) for an empirical investigation of the implications of our model for returns.

In order to compute the expected rate of return of the different assets it is useful to consider first the pricing kernel process,

$$
\frac{d m_{t}}{m_{t}}=-\mu_{m}\left(S_{t}\right) d t+\sigma_{m}\left(S_{t}\right) d \mathbf{B}_{t} \quad \text { where } \quad m_{t}=u_{c}\left(C_{t}, X_{t}, t\right)=e^{-\phi t}\left(\frac{Y_{t}}{C_{t}}\right)
$$

and

$$
\begin{aligned}
& \mu_{m}\left(S_{t}\right)=\phi+\mu_{c}-\sigma_{c} \sigma_{c}^{\prime}+k\left(1-\bar{Y} S_{t}\right)-\alpha\left(1-\lambda S_{t}\right) \sigma_{c} \sigma_{c}^{\prime} \\
& \sigma_{m}\left(S_{t}\right)=-\left[1+\alpha\left(1-\lambda S_{t}\right)\right] \sigma_{c} .
\end{aligned}
$$

Expression (24) is essential in the computation of the expected excess rate of return of any asset, which is the instantaneous covariance between the increments in returns and the kernel, $\sigma_{R, t}^{i} \sigma_{m}^{\prime}$ (see, for example, Duffie (1992), page 98.) 


\section{IV.A The risk free rate}

The risk free rate is given by $\mu_{m}\left(S_{t}\right)$, that is,

$$
r^{f}\left(S_{t}\right)=\phi+\mu_{c}-\sigma_{c} \sigma_{c}^{\prime}+k\left(1-\bar{Y} S_{t}\right)-\alpha\left(1-\lambda S_{t}\right) \sigma_{c} \sigma_{c}^{\prime}
$$

a version of the formula found in Campbell and Cocrane (1999). The interpretation of this formula is standard (see Campbell, Lo, and MacKinlay (1997), page 331, equation 8.4.19.)

\section{IV.B The return on the total wealth portfolio}

The excess return on the total wealth portfolio is

$$
d R_{t}^{T W}=\frac{d P_{t}^{T W}+C_{t} d t}{P_{t}^{T W}}-r^{f}\left(S_{t}\right) .
$$

An application of Ito's Lemma and the equilibrium condition $\mu_{R, t}^{T W}=-\sigma_{R}^{T W}\left(S_{t}\right) \sigma_{m}^{\prime}\left(S_{t}\right)$ allows us to write the dynamics of the return of the market portfolio as:

$$
d R_{t}^{T W}=\mu_{R}^{T W}\left(S_{t}\right) d t+\sigma_{R}^{T W}\left(S_{t}\right) d \mathbf{B}_{t}
$$

where

$$
\begin{aligned}
\mu_{R}^{T W}\left(S_{t}\right) & =\left(1+\alpha\left(1-\lambda S_{t}\right)\right)\left[1+\frac{k \bar{Y} S_{t}\left(1-\lambda S_{t}\right) \alpha}{k \bar{Y} S_{t}+\phi}\right] \sigma_{c} \sigma_{c}^{\prime} \\
\sigma_{R}^{T W}\left(S_{t}\right) & =\left[1+\frac{k \bar{Y} S_{t}\left(1-\lambda S_{t}\right) \alpha}{k \bar{Y} S_{t}+\phi}\right] \sigma_{c}
\end{aligned}
$$

To understand the intuition, consider first the case where consumption is far away from habit, that is $S_{t}=\lambda^{-1}$. In this case, risk-aversion is low and expected returns and variance are low, both equal to $\sigma_{c} \sigma_{c}^{\prime}$. As $S_{t}$ decreases, current consumption gets closer to the habit level, which in turn increases both the degree of risk aversion and the volatility of the returns on the total wealth portfolio. These two effects contribute to increase the premium investors require to hold stocks. As $S_{t}$ gets close to zero, however, the volatility of the surplus also declines to zero, because $S_{t}$ is a process in $\left[0, \lambda^{-1}\right]$. This latter property carries over to the volatility of returns, that declines when $S_{t}$ approaches zero, partially undoing the effect of the increase in the degree of risk aversion. When this effect is large enough the required premium falls.

However, this effect does not apply to the Sharpe ratio, which is always decreasing in $S_{t}$ :

$$
\operatorname{SR}\left(S_{t}\right)=\frac{\mu_{R, t}^{T W}\left(S_{t}\right)}{\sqrt{\sigma_{R}^{T W}\left(S_{t}\right) \sigma_{R}^{T W}\left(S_{t}\right)^{\prime}}}=\left(1+\alpha\left(1-\lambda S_{t}\right)\right) \sqrt{\sigma_{c} \sigma_{c}^{\prime}} .
$$


The Sharpe ratio then moves countercyclically, decreasing in booms and raising in recessions. The Sharpe ratio plays an important role in the interpretation of the formulas for the returns of individual securities below.

To synthesize the properties of the aggregate variables in this model, and to compare them to the model of Campbell and Cochrane (1999), Panels A-D of Figure 3 plot some of the aggregate variables against the level of the surplus $S_{t}$. The plots use calibrated parameters, as

discussed in section V. Panel A plots the stationary density of the surplus-consumption ratio $S_{t}$, given the calibrated parameters. Panel B plots the price-consumption ratio of the total wealth portfolio, which has the characteristic upward slope with respect to $S_{t}$. Panel C plots the excess expected return and volatility of the total wealth portfolio, along with the real rate of interest. Finally, Panel D plots the Sharpe-ratio of the total wealth portfolio, as function of the state variable $S_{t}$. Comparison with the corresponding Figures in Campbell and Cochrane (1999, see Figures 2 to 6, pages 219-223) reveals essentially the same qualitative behavior of all these functions. The only difference is in the expected returns and volatility functions, as they bend back towards zero as $S_{t}$ gets close to zero. However, the region of $S_{t}$ where these function are upward sloping has a very small unconditional probability, as shown by the stationary density of $S_{t}$ in Panel A.

\section{IV.C The return on individual securities}

We now turn to individual securities. Again, it is useful to first compute the return processes for the two special cases introduced in Section III.

\section{IV.C.1 Model A: Returns in the absence of habit persistence}

We start by finding the expected excess rate of return in the absence of habit persistence effects. First recall that in the standard log economy the expected excess rate of return of the total wealth portfolio is simply given by $\sigma_{c} \sigma_{c}^{\prime}$. Then the next propositions characterizes the basic moments of the returns of individual securities.

Proposition 5. (Expected returns under Model $A$ ) Let $\alpha=0$ and $Y_{t}=\bar{Y}=\lambda=1$, then the expected excess rate of return of asset $i, \mu_{R, t}^{A, i}$, is given by

$$
\mu_{R, t}^{A, i}=\sigma_{c} \sigma_{c}^{\prime}+\frac{\phi s_{t}^{i}}{\phi s_{t}^{i}+\kappa \bar{s}^{i}}\left(\theta^{i}-\sum_{j=1}^{n} s_{t}^{j} \theta^{j}\right) .
$$


Assets whose shares covary positively with consumption growth, so that $\theta^{i}-\sum_{j=1}^{n} s_{t}^{j} \theta^{j}>$ 0 , will command a higher premium than the total wealth portfolio, as the asset provides little insurance against adverse consumption shocks. This is more so as $s_{t}^{i}$ increases, as the asset will make a larger fraction of consumption. Clearly, the opposite argument holds for the case where the dividend-consumption share covaries negatively with consumption growth.

IV.C.2 Model B: Returns in the absence of cash flow effects

When habit persistence is present but there are no cash flow effects, there can be no cross sectional dispersion in expected returns in the long run and all the variation observed in sample is solely due to conditional cross sectional variation. This conditional cross sectional variation is driven by shocks to cash flows but these shocks have rather different effect on the cross section depending on when they occur over the business cycle.

Proposition 6. (Expected returns under Model B.) Let $\operatorname{cov} t\left(\frac{d D_{t}^{i}}{D_{t}^{i}}, \frac{d C_{t}}{C_{t}}\right)=\sigma_{c} \sigma_{c}^{\prime}$, then the expected excess rate of return of asset $i, \mu_{R, t}^{B, i}$, is given by

$$
\mu_{R, t}^{B, i}=\left(1+\alpha\left(1-\lambda S_{t}\right)\right)\left[1+\frac{k \bar{Y} S_{t} \alpha\left(1-\lambda S_{t}\right)}{k \bar{Y} S_{t}+\left(1+f\left(s_{t}^{i} / \bar{s}^{i}\right)\right) \phi}\right] \sigma_{c} \sigma_{c}^{\prime}
$$

where

$$
f\left(\frac{s_{t}^{i}}{\bar{s}^{i}}\right)=\frac{(\phi+k) \kappa\left(\frac{s_{t}^{i}}{\bar{s}^{i}}-1\right)}{(\phi+\kappa) \kappa+(\phi+k)\left(\kappa+\phi \frac{s_{t}^{i}}{\bar{s}^{i}}\right)} .
$$

It follows from equation (31) that $f\left(s_{t}^{i} / \bar{s}^{i}\right) \gtrless 0$ whenever $s_{t}^{i} \gtrless \bar{s}^{i}$, and that $f(1)=0$ when $s_{t}^{i}=\bar{s}^{i}$. Then one can easily check that,

$$
\mu_{R, t}^{B, i} \gtreqless \mu_{R, t}^{T W} \quad \text { iff } \quad s_{t}^{i} \lesseqgtr \bar{s}^{i}
$$

where $\mu_{R, t}^{T W}$ is given by equation (26).

To understand the intuition behind (32) take the case where $s_{t}^{i}<\bar{s}^{i}$. In this case the dividends of asset $i$ are likely to experience growth above that of consumption and this effect gets incorporated into current prices. Still, the conditional expected excess returns are above that of the total wealth portfolio. At this stage it is useful to return to the price of asset $i$ under Model B, given in expression (18) and that we can rewrite as

$$
P_{t}^{B, i}=\bar{s}^{i}\left[P_{t}^{T W}+\left(\frac{s_{t}^{i}}{\bar{s}^{i}}-1\right)\left(1+\frac{k}{\phi+\kappa}\left(\frac{S_{t}}{\bar{S}}\right)\right) \frac{C_{t}}{\phi+\kappa+k}\right]
$$


where $P_{t}^{T W}$ is given in equation (11). When $s_{t}^{i}<\bar{s}^{i}$ the second term in parenthesis becomes negative and hence the relative impact of changes in the level of $P_{t}^{T W}$ on $P_{t}^{i}$ increases. This decreases the diversification opportunities offered by asset $i$ and hence increases its required premium to hold it. The second effect is a "dampening" or "hedging" effect: If $s_{t}^{i}<\bar{s}^{i}$ then positive shocks to $C_{t}$, and hence to $S_{t}$, translate into negative shocks of the second term in parenthesis, which partly counterbalance the positive movement in $P_{t}^{T W}$ induced by the same shocks. It can be shown though that the first "level" effect dominates the "hedging" one.

\section{IV.C.3 Returns in the general case}

We use our approximate pricing formula to obtain the expected excess rate of return of individual assets in the general case. The formula for returns simply combines the two sources discussed in the previous two subsections, as it is given by

$$
\mu_{R, t}^{i} \approx\left(1+\alpha\left(1-\lambda S_{t}\right)\right)\left[\left(1+\gamma_{1}\left(s_{t}^{i}, S_{t}\right) S_{t}\left(1-\lambda S_{t}\right) \alpha\right)+\gamma_{2}\left(s_{t}^{i}, S_{t}\right) s_{t}^{i} \theta^{i}\right] \sigma_{c} \sigma_{c}^{\prime}
$$

where the functions $\gamma_{1}\left(s_{t}^{i}, S_{t}\right)>0$ and $\gamma_{2}\left(s_{t}^{i}, S_{t}\right)>0$ are given in equations (39) and (40) in Appendix B.

Briefly, the first term in the product, $\left(1+\alpha\left(1-\lambda S_{t}\right)\right)$, is proportional to the Sharpe ratio of the total wealth portfolio and, naturally, it is common across assets. When the Sharpe ratio is high, which occurs when the surplus consumption ratio is low, this term is high and as a consequence so will be the expected excess rate of return. The first term in brackets is related to the covariation of the marginal rate of substitution with changes in the surplus consumption ratio, and it is similar in intuition to the one in discussed in Section IV.C.2 (Model B). Instead, the second term in the bracket captures the component of the premium that results from the covariation of the marginal rate of substitution with the asset's cash flow shocks, and it is similar to the one in discussed in Section IV.C.1 (Model A). ${ }^{12}$

\section{CALIBRATION}

\section{V.A Data}

We calculated quarterly dividends, returns, market equity and other financial series from the CRSP database. We use CRSP and COMPUSTAT data files to form 20 value-weighted

\footnotetext{
${ }^{12}$ Notice that under condition $(5)$, which we invoke when we look at the general case, we have that $\mu_{R, t}^{A, i} \approx$ $\sigma_{c} \sigma_{c}^{\prime}+\frac{\phi s_{t}^{i}}{\phi s_{t}^{i}+\kappa \bar{s}^{i}} \theta^{i}$.
} 
industry every month formed according to their two digit Standard Industrial Classification (SIC) code. ${ }^{13}$ Finally, whenever possible, we augmented our financial series with accounting data from the Compustat data base. To merge the two files we rely on the link structure issued by CRSP. Table II provides a description of the industry portfolio and summary statistics.

The real consumption data is obtained from the National Income and Product Accounts (NIPA) for the period 1946-2001. As many before us, we define total consumption as nondurables plus services. We use the Shiller (1989) annual data for the period 1927-1945, where we interpolate the consumption data to obtain quartely quantities. ${ }^{14}$ The risk free rate is the 90-day Treasury bill and the inflation rate is calculated using the Consumer Price Index. ${ }^{15}$

\section{V.B Model parameters}

\section{V.B.1 Preference parameters, $\{\phi, k, \bar{Y}, \lambda, \alpha\}$}

We use the stationary distribution of the process $Y_{t}$, given in equation (36) in the appendix, to calibrate the preference parameters, $\{\phi, k, \bar{Y}, \lambda, \alpha\}$, to the aggregate market portfolio and interest rates. For a given choice of parameters, we can then compute the population averages of expected returns, return volatility, and price-consumption ratio for the total wealth portfolio, and the average level and volatility of risk-free rate. We match the population averages for the total wealth portfolio with the corresponding values for the market portfolio in the data. Clearly the total wealth portfolio and the market portfolio are different but our results show that they are very similar in terms of moments and prices. For this reason we use the total wealth portfolio as we have convenient formulas for the returns and prices that can be easily used to match them to data. ${ }^{16}$

\footnotetext{
${ }^{13}$ The two digit SIC grouping are similar to those employed by Moskowitz and Grinblatt (1999) and Boudoukh, Richardson and Whitelaw (1994). The SIC codes are obtained from CRSP, which reports the time-series of industry classification codes. Although the COMPUSTAT classification is considered to be more accurate, the series is modified only from 1994, which leads to a survival bias.

${ }^{14} \mathrm{We}$ also tested our results in the period starting in 1963 to reflect the concerns of some authors that the sample of firms in COMPUSTAT is limited in the earlier part of the sample. See Fama and French (1997, page 156.) This is also the sample period chosen by Moskowitz and Grinblatt (1999). In addition, we also used the sample from 1953:1 to 1999, favored by some as it avoids the Korean war. See, among others, Campbell and Mankiw (1990). The results were very similar and are not reported here.

${ }^{15}$ The real rate has been computed as $r_{R, t}=r_{N, t}-E_{t}\left[\right.$ inflation $\left._{t}\right]$, where the latter is obtained from fitting an $\mathrm{AR}(4)$ to the inflation process.

${ }^{16}$ That the distinction between the total wealth portfolio and the market portfolio is less relevant than may seem at first is also to be found in Campbell and Cochrane (1999), see in particular their comments in page 221.
} 
In our model $\lambda$ and $\bar{Y}$ cannot be identified separately: All our pricing implications depend on the ratios $\bar{Y} / Y_{t}$ and $\lambda / Y_{t}$. Given the linearity of the process (7), if we scale $\bar{Y}, \lambda$ and $Y_{t}$ by a constant, no changes will occur to the prices, returns or interest rates. Without any loss of generality then we choose to set the average inverse surplus to match the steady state degree of risk aversion in Campbell and Cochrane (1999), $\bar{Y}=29.6 .{ }^{17}$ The remaining parameters $\{\phi, k, \lambda, \alpha\}$ are then obtained by matching (i) the average excess stock return, (ii) the average interest rate, (iii) the interest rate volatility, (iv), the average price consumption ratio and (v) the average Sharpe ratio. The parameter values so obtained are in Panel A of Table III, while the population averages of the total-wealth portfolio, as well as the sample averages in the 1927-2001 periods are in Table IV. The calibrated model succeeds in matching the equity premium, the average price-dividend ratio, the volatility of market returns, and the Sharpe ratio. The model also delivers the right level and volatility of the risk free rate.

\section{V.B.2 Share process parameters, $\left\{\kappa, \bar{s}^{i}, \mathbf{v}^{i}\right\}_{i=1, \cdots, n}$}

The share process (4) presents several challenges for the estimation. A full analysis of the estimation procedure is outside the scope of this paper and we leave it to future research. Here we outline a simple estimation procedure, which relies on an approximation of the share process that is similar to the one we used to obtain prices of individual assets in the general case. Monte Carlo simulations show that this methodology works well when $n$ is "large" and the variance of the share process is not too large. Appendix B contains additional details.

The methodology is in two steps: First, we estimate the drift parameters $\left\{\kappa, \bar{s}^{1}, . ., \bar{s}^{n}\right\}$ through GMM applied to the Euler discretization of the process (4). Since by assumption the process for shares has a stochastic volatility, we use a two-stage GMM with NW corrected variance covariance matrix. The second steps is to compute the vectors $\left\{\mathbf{v}^{1}, . ., \mathbf{v}^{n}\right\}$. To do this, we assume that the term $\sum_{j=1}^{n} s_{t}^{j} \mathbf{v}^{j}$ that appears in the volatility function in (4) is approximately constant. In this case, we can back out the vectors $\mathbf{v}^{i}$ 's from the variancecovariance matrix of the process for the $\log s_{t}^{i}$. The results of the estimation are contained

\footnotetext{
${ }^{17}$ This value of $\bar{Y}$ is slightly higher than the one in Campbell and Cochrane (1999). The steady state surplus consumption ratio these authors is $\bar{S}=.0676$. Given that they work with $\gamma=2$ rather than with $\gamma=1$, their steady state degree of risk aversion is $\frac{\gamma}{\bar{S}} \approx 29.6$. The difference is due to the fact that our results match the numerical average in our generated Campbell and Cochrane (1999) surplus consumption series. Clearly, the choice of $\lambda$ affects the volatility of the habit and this prevents setting it equal to its perhaps natural boundary of one as this would induce, contrary to intuition, excessive volatility in the habit.
} 
in Panel B of Table III. We do not report the entire matrix $\mathbf{v}=\left(\mathbf{v}^{1}, \ldots, \mathbf{v}^{n}\right)^{\prime}$, but only the coefficients that are relevant for pricing, namely, $\theta^{i}=\mathbf{v}^{i} \sigma_{c}^{\prime}$.

\section{MODEL EVALUATION}

To evaluate the model we conduct two types of excercises. First, in Section VI.A we run simulations of our economy. This will allow us to compute moments that are of interest and for which we have no closed form solutions. Then in Section VI.B we evaluate whether our model can account for the observed financial history of the US. To accomplish this we feed the formulas found in Section III the realized consumption and cash flow shocks and ask whether the calibrated time series can reproduce the main patterns observed in the data.

\section{VI.A Performance of the model in simulated data}

We simulate 10,000 time series of length equal to the available time series. To ease the comparison with data, we simulate consumption and shares at very small intervals (1 day) but retain information only at the quarterly frequency. This generates a number of quarters slightly above the two million mark. The simulations employ the parameter values reported in Section V. In order not to bias the simulation procedure, each simulation includes an initial "burn out" period so each simulation starts in a randomly selected point.

\section{VI.A.1 Means and standard deviations}

Panel A of Table IV reports some summary statistics from the simulations for aggregate variables, namely, the market portfolio and the interest rate. As already mentioned, the model is sufficiently flexible to match well the sample moments of the market portfolio. In particular the Sharpe ratio is matched almost to the point. In addition the volatility of market returns, a moment that was not used to calibrate the model, is close to that observed in the data. The model succeeds as well in reproducing the level and volatility of the risk free rate, a standard difficulty in habit persistence models. The model is then able to reproduce the results of Campbell and Cochrane (1999) for the market portfolio.

Importantly the calibration did not use any cross sectional information and it is here that the validity of the model can start to be objectively assessed. Panel B reports some summary statistics about the cross-section of prices and returns of industry portfolios. For ease of comparison with the actual realized averages, Figure 4 plots the main results contained in Table IV Panel B. Panel A in Figure 4 plots the the sample average excess return for each 
industry versus the mean obtained of our simulation. The model is able to generate average excess returns across industries that are close to those observed in the data though the sample average is slightly above the average return obtained across simulations. This is to be expected, given that the same occurs at the market portfolio level.

Panel B plots the sample average price-dividend ratios versus the average price dividend ratio in the simulated data. Once again the model reproduces its sample counterpart with the only exception of Construction, where the model generates an average price dividend ratio well out of line with data. Finally, Panel C reports the sample average Sharpe ratios versus their simulations counterpart, and once again the model performs well. The model is then able to replicate the trade-off between risk and return across industries.

\section{VI.A.2 Predictability in the aggregate and in the individual industries}

Table V contains the results from standard predictability regressions, both in the data and in the simulations. Panel A reports results for the aggregate market portfolio. For completeness, and to ease the comparison with previous research, we report the predictability regressions for two samples periods, the full sample 1927-2001 and for the shorter and most standard postwar sample 1947-1994. Similarly to Campbell and Cochrane, who also use a long and short sample though both ending in 1995, the model produces a good deal of predictability at the aggregate level. The model successfully reproduces the increasing nature of $\mathrm{R}^{2} \mathrm{~s}$ versus the horizon. For example, the average $\mathrm{R}^{2}$ across simulations in a two year ahead predictability regression is $17.7 \%$, against a $6 \%$ in the full sample and $29 \%$ in the post-war sample. At the 5 year horizon, the numbers are $26.7 \%$ in average across simulations, and $12 \%$ and $56 \%$ in the long and short sample respectively. Importantly the predictability in the short sample is stronger than in the 1927-2001 one as the former excludes the period 1995-2001 that saw high price dividend ratios and high realized returns.

Panel B reports the predictability regressions for individual industries, which, as before, can be more easily grasped graphically. Figure 5 Panel A shows that, consistently with the data corresponding to the long sample period, the model produces a large cross-sectional difference in the two and five year ahead predictability of returns, though it slightly overestimates the two year predictability. The model successfully reproduces the low predictability for industries

such as Mining, Construction, and Railroads and the high predictability for Chemical and Utilities. The model does also well for industries with an intermediate level of predictability 
like Dept. Stores. Similar results obtain for the five year predictability (Figure 5 Panel B.)

How are we to interpret this cross sectional variation within the context of our model? Predictability at the aggregate level is exclusively driven by time-variation in the surplusconsumption ratio $S_{t}$, which affects the stochastic discount factor. In the case of individual assets though, to the impact of the time variation in the stochastic discount factor we have to add the fluctuations of share $s_{t}^{i}$ around its long run mean $\bar{s}^{i}$, as equation (33) shows. This additional effect is purely idiosyncratic and it induces differences in the extent to which price dividend ratios can predict future returns across industries. To isolate this cash flow component consider, for example, Model A where the variation in the stochastic discount factor was shut down. Then, by simple manipulation of formula (29) we find

$$
\mu_{R, t}^{A, i} \approx \sigma_{c} \sigma_{c}^{\prime}+(\phi+\kappa) \theta^{i} \frac{D_{t}^{i}}{P_{t}^{i}}
$$

where we have imposed that, approximately, $\sum_{j=1}^{n} s_{t}^{j} \theta^{j} \approx 0$ (see condition (5)). The level of $\theta^{i}$ determines the level of predictability in the absence of discount factor effects. In such a model, expected returns change as the covariance between consumption and returns is regulated by the shares, $s_{t}^{i}$, and hence by the dividend price ratio. When there are discount factor effects, the interaction between the cash flows and the discount factor becomes hard to disentangle and a high $\theta^{i}$ may even result in less predictability. For example, suppose that a stock's share is positively correlated with consumption. Then $s_{t}^{i}$ will, on average, decrease with $S_{t}$. The effect on the price-dividend ratio is now ambiguous. On the one hand, a lower $S_{t}$ results in a lower price dividend ratio as the investor has become more risk averse. On the other, the lower $s_{t}^{i}$ will tend to push the price dividend ratio up, as dividend growth above that of consumption is expected. Notice though that both effects unambiguously contribute to the increase in expected returns.

Another factor that affects the extent to which industry returns are predictable is the volatility of cash flows, determined by $\mathbf{v}^{i} \mathbf{v}^{i^{\prime}}$. Clearly, other things equal, the higher this volatility the lower the predictability as a larger component of the variation in returns is driven by shocks to cash flows, a point to which we return in the next section. Finally, assets differ in one additional dimension and it is in their long run contribution to the overall consumption, $\bar{s}^{i}$. Provided that $s_{t}^{i}$ is close to its long run mean, the higher the value of $\bar{s}^{i}$ the closer the asset is to being effectively the market portfolio and hence the higher the predictability of returns 
of asset $i .^{18}$

The next section uses standard price-dividend ratio decompositions applied to industry portfolios in order to shed some additional light on the predictability results.

\section{VI.A.3 The variance decomposition: news to cash flows and news to expected returns}

Variation in price dividend ratios of individual securities is driven by both changes in the forecasts of dividend growth and changes in the forecasts of future discount rates, the two building blocks of our model. To asses how much of the variance of the price dividend ratio of individual industry portfolios is driven by each component in the data we can use the variance decomposition of the (log) price dividend ratio proposed by Cochrane (1992),

$$
\begin{aligned}
\operatorname{var}\left(\log \left(P_{t}^{i} / D_{t}^{i}\right)\right) \approx & \sum_{j=1}^{\infty} \rho^{j} \operatorname{cov}\left(\log \left(P_{t}^{i} / D_{t}^{i}\right), \Delta \log \left(D_{t+j}^{i}\right)\right) \\
& -\sum_{j=1}^{\infty} \rho^{j} \operatorname{cov}\left(\log \left(P_{t}^{i} / D_{t}^{i}\right), \log \left(R_{t+j}^{i}\right)\right),
\end{aligned}
$$

and then compare these sample estimates with their counterparts in the simulations. As argued in the previous section this exercise will throw light on the cross sectional differences in the predictability regressions.

Table VI contains the results of this variance decomposition. Recall that the decomposition is not an orthogonal one so quantities higher than $100 \%$ and less than $0 \%$ are possible. Panel A contains the results at the aggregate level and it shows that the model matches the data to a good degree: Essentially, most of the variation in the price-dividend ratio in the model (at the aggregate level) stems from changes in returns, that is, changes in the stochastic discount factor.

Panel B contains the same variance decomposition across industries which are more conveniently presented in Figure 6. The return component makes for a large part of the variance of price dividend ratios for many of the industries in our sample. In particular, it does so strongly for Paper, Chemical, Petroleum, Construction, Machinery, Electrical Eq. and Utilities. Mining, Food and Retail on the other hand seems to be equally driven by each

\footnotetext{
${ }^{18}$ To confirm these intuitions, in results not reported here, we regressed the predictability obtained in the model, as measured by the $\mathrm{R}^{2}$ of the two and five year predictability regressions for each industry, on the three parameters that completely determine the cash flow process for each asset, $\mathbf{v}^{i} \mathbf{v}^{i^{\prime}}, \theta^{i}$, and $\bar{s}^{i}$. The three parameters came strongly significant with the expected signs, and explained more than $85 \%$ of the variation in the cross-sectional differences in average $\mathrm{R}^{2}$ across simulations.
} 
component. Finally, the variance of the price dividend ratio of Prim. Metals and Railroads is dominated by its cash flow component.

The model also produces considerable cross-sectional differences in the variance decomposition across industries, and for most industries it replicates the empirical counterpart to a good extent. For example, as in the data, the model also predicts that the price-dividend ratios of Chemicals, Petroleum and Utilities should be mainly driven by shocks to returns, the ones of Prim. Metals and Railroads should be mainly driven by shocks to cash flows, and the one of Food should be equally driven by the two sources. For other industries, however, the model seems to slightly underpredict the fraction of the variance of the price dividend ratio that is driven by the return component and slightly overpredict the fraction that is driven by cash flow shocks. Thus, for a number of industries, such as Machinery, Electrical Eq. and Paper, the model predicts a more equal weight across the two sources of variation in price-dividend ratios than the data imply. Finally, for some industries, such as Construction, Apparel and Paper, the model's predictions are not reflected in the data estimates. Yet, the model succedes in producing large cross-sectional differences in the sensitivity of the price-dividend ratio to cash flow shocks and return shocks.

The intuition for this result can be grasped by looking at equation (20). After simple manipulations, we can write the price-dividend ratio as $P_{t}^{i} / D_{t}^{i} \approx \psi_{0}^{i}\left(S_{t}\right)+\psi_{1}^{i}\left(S_{t}\right)\left(\bar{s}^{i} / s_{t}^{i}\right)$, for two functions $\psi_{0}^{i}\left(S_{t}\right)$ and $\psi_{1}^{i}\left(S_{t}\right)$. For typical parameter values we have $\partial \psi_{1}^{i}(S) / \partial S>0$. Consider now an asset $i$ characterized by a relatively low volatility of $s_{t}^{i}$. In this case, most of the variation of the price-dividend ratio stems from changes in $S_{t}$, which is related to aggregate returns. Thus, a variance decomposition would reveal a large return component. Conversely, an asset characterized by a large volatility of the share $s_{t}^{i}$ would have a price-dividend ratio greatly affected by the movement in $s_{t}^{i}$. Thus, the variation of price-dividend ratios would be mainly affected by cash flow shocks. ${ }^{19}$ This intuition nicely links our empirical results with those of Vuolteenaho (2002). Indeed, he finds that firm-level returns are mainly driven by cash flow shocks, and that these cash flow news are essentially idiosyncratic. Within the context of our model, we can see that at the very disaggregate level, the (idiosyncratic) volatility of shares

\footnotetext{
${ }^{19}$ As for the predictability case, we verified this intuition by regressing cross-sectionally the average (acrosssimulations) cash-flow component and return component of the variance of the $P / D$ ratios on the various characteristics of the cash-flow process. The volatility $\mathbf{v}^{i} \mathbf{v}^{i \prime}$ is by far the most important driving force of the cross-sectional differences, with the signs as explained in the text.
} 
would be very high, generating most of the variance of the price-dividend ratio. In the case of industries, instead, aggregation reduces the (idiosyncratic) volatility of shares, increasing then the role of returns in the variance decomposition. In the limit, at the aggregate market level, cash flow news have little effect and only returns affect the price-dividend ratio.

\section{VI.B The historical performance of the US stock market}

Inspection of equation (20), together with equations (14) and (18), highlights the obvious: The model makes point by point predictions of what the price should have been given the realized fundamentals. If there is any discrepancy between the historical price and the one implied by the model then this can be immediately rejected. Still, does our model reproduce the main patterns observed in the historical experience of the US stock market? To answer this question, we feed the pricing formulas in Section III.B.3 the observed consumption and cash flow shocks and see whether the resulting prices match the observed patterns.

\section{VI.B.1 Aggregate quantities}

Figure 7 Panel A plots the surplus consumption ratio implied by the model once we feed to the estimated model the observed consumption shocks. Panel A also includes the surplus consumption ratio of Campbell and Cochrane (1999) for comparison's sake. The two series track each other tightly. The surplus consumption ratio captures the main patterns of the US business cycle: The depression of the 30s (with $S_{t}$ hitting almost zero), the 1960's expansion, the recession in the mid 70's and early 80's, the recovery afterwards, the mild recession of the early 90's and the strong expansion that followed it. It also shows the start of the new recession at the end of 2001. Panel B includes the consumption series together with the habit level, $X_{t}$, implied by our surplus consumption series. As intuition suggests, the habit level moves slowly and tracks consumption.

Panel $\mathrm{C}$ of Figure 7 reports the plot of the time-series of market portfolio log pricedividend ratio in the data and the one generated by the model. As found by Campbell and Cochrane (1999), the model offers a remarkable account of the realized history of the log price dividend ratio of the market portfolio. Still there are two interesting periods in which the model generated price dividend ratio does not track the realized one. First, the model is not able to replicate the extraordinary performance of the market in the second half of the 90s, a usual shortcoming. There are two possible explanations for this. First, the percentage of firms that switched from dividend distribution to share repurchases as a mean of distributing 
earnings increased considerably in the 90s (see Fama and French (2002)). If this is the case, cash distributions would be seriously underestimated during this period and as a consequence the price dividend ratio would appear higher than its actual level. An alternative explanation, one favoured by many, is that the nineties witnessed a phenomenal speculative bubble, a possibility our model does not allow for. Further research is then needed to account for the performance of the market in the 90 s.

Second, the model also predicts price dividend ratios that are lower than the realized ones during most of the 30s. The reason is simple and it stems from the high volatility of consumption growth during the $30 \mathrm{~s}$, which led the surplus-consumption ratio $S_{t}$ to hit almost zero. From Panel A of Figure 3, the stationary distribution of $S_{t}$ shows that this is an almost zero-probability event and it stems from a slight mispecification of the model. Indeed, in order to account for this behavior, we should assume that consumption growth has a timevarying volatility, which we have ruled out in our assumption that consumption growth is i.i.d. Although the model implies an unrealistically low price dividend ratio during this period, we prefer to keep the model simple rather than construct an even more complicated model to account for the 30s.

\section{VI.B.2 The price-consumption ratios across industries}

Figures 8 and 9 plot the fitted and the actual price-consumption ratios for the 20 industries in our sample. The reason for concentrating on the price consumption ratios, rather than on price dividend ratios, is that we want to asses whether the model can account for the observed levels of prices, and it is best to normalize them by a common factor like consumption in order to make them stationary. Dividing each industry's price by its corresponding dividend obscures the comparison as dividend measurement error may be different across industries. Still, price dividend ratio plots are very similar to the price consumption ratio ones.

As we can see, the model implied price consumption ratios track well their empirical counterparts. For instance, the fitted time series account for the secular decline of Railroads and Primary Metals and the steady rise of Financials. It also explains the main fluctuations in Chemical, Fab. Metals, Food, Paper, Machinery, Electric Eq., Retail, and Others. The

model seems to be less successful in explaining the time series of Construction, Utilities or Transportation Equipment.

Notice also that, interestingly enough, price consumption ratios for some industries saw 
remarkable levels during the late 60s and early 70s. For instance, Manufacturing, Apparel, and Other Transportation saw levels of price consumption ratios during that period that have no equivalent at any other point in time. Our model captures the rise in the price consumption ratio of Apparel and partly the one of Other Transportation, but it misses the one in Manufacturing. ${ }^{20}$

To better understand the differences between all these time series it is illustrative to concentrate on two industries for which the model seems to perform well: Paper and Railroads. Panels A and B in Figure 10 reproduce the price consumption ratios, both fitted and realized, for these two industries. Panels $\mathrm{C}$ and $\mathrm{D}$ report the two shares that correspond to these two industries. First notice the long decline that Railroads has experienced during our sample period. The decline in its price consumption ratio mirrors the decreasing importance of this sector in the overall market. The price consumption ratio held relatively well during the 1960s, precisely when the share decline temporarily halted and even rose slightly. Importantly, the time series pattern of the price consumption ratio of Railroads seems dominated by its idiosyncratic component: The precipitous drop of its share over consumption. Business cycle components seem less important and the price consumption ratio does not react to either the mid seventies or early eighties recession.

Paper shows a rather different pattern. First, the share of Paper experienced an important increase in the decade following WWII and remained fairly constant after that, perhaps showing early hints of a recent decline. The price consumption ratio mirrored these developments but with a certain lag, due to the fact that the economy experienced a downturn after the conflict that undid the positive effect of the share increase in the price. Indeed notice that $S_{t}$ declined considerably after 1945-6. It is then only in the fifties that the price consumption ratio of Paper "catches up" with its share's increase. ${ }^{21}$ The trend continued during the next

\footnotetext{
${ }^{20}$ The sudden rise of the price consumption ratio during those years seems largely to be restricted to these three industries, whereas the rise in the 90s happens in ten of the twenty industries. Of these three industries only Apparel and Other Transport. saw a slight rise and then decline in their shares (these plots are not reported,) which can account for the qualitatively aspect of the pattern but certainly not enough quantitatively. The share of Manufacturing saw a steady increase throughout that period.

${ }^{21}$ At this point it is illustrative to compare our model with the traditional Gordon model. The latter implies a price consumption ratio that is proportional to $s_{t}^{i}$. For this reason the Gordon model would have the price consumption ratio of Paper to immediately react to the increase in the share. In contrast our model manages to reproduce the lag as it allows for the countervailing effect of the decrease in the surplus consumption ratio.
} 
two decades, when the surplus consumption ratio was on the rise, but it was cut when the recession in the mid seventies kicked in and that continued all the way to early eighties, when the worst recession in the post-war period set in. Paper recuperated after that and benefited from the increase in the surplus consumption ratio that now undoes the effect of a declining share on the price consumption ratio.

In summary, as presented in section III, prices are driven by both idiosyncratic components and aggregate ones, and their importance is rather different depending on the particular industry. The model and its calibration are able to capture a crucial component in the diverse historical experiences present in the cross section.

\section{VI.B.3 The time series of the cross sectional standard deviation of asset prices}

Figure 11 plots the cross-sectional dispersion of price-consumption ratios (Panel A) and price-dividend ratios (Panel B), and their empirical counterpart. ${ }^{22}$ The plots have a number of implications: First, Panel A shows that the cross-sectional dispersion of asset prices is, to a first degree, generated by dividend levels. This follows from the fact that basic manipulations of the formulas found in Section III.B.3 show that the price consumption ratio of an individual industry is linear in the share, $s_{t}^{i}$, although with stochastic coefficients. Second, a comparison of the fitted plot with the dynamics of the surplus $S_{t}$ in the top panel of Figure 7 reveals that the standard deviation of price consumption ratios drops when $S_{t}$ does as well.

Panel B shows the cross-sectional standard deviation of price-dividend ratios. Also in this case the model produces the correct level of dispersion and the correct dynamics: Interestingly, while the cross-sectional dispersion of price dividend ratios increased slowly in the $50 \mathrm{~s}$ and $60 \mathrm{~s}$ to drop in the 70s, our model produced a much quicker increase in dispersion in the middle of the $50 \mathrm{~s}$, and steady decline until the end of $70 \mathrm{~s}$, where it matched again the level of the crosssectional dispersion of price dividend ratios in the data. Then, both series increase, although the 90 s saw the dispersion in the data surge considerably.

Table VII shows the correlation between the time-series of the market price dividend ratio and the time-series of the cross-sectional dispersions of the price dividend ratios of individual assets both in the data and the simulations. The model successfully accounts for a large

Of course, the Gordon model can be easily rejected as it does not allow for time varying price dividend ratios.

${ }^{22}$ Differently from Figure 1, we plot the cross-sectional standard deviation of the level of price-consumption ratios and price-dividend ratios, as we are interested in seeing the fit against their empirical counterparts, and not the correlation with the market price consumption ratio. 
percentage of the correlation though not all. Specifically, while the model produces an average correlation (across simulations) between the market log price dividend ratio and the crosssectional dispersion of individual price dividend ratios of about $23 \%$, the data show a higher number, about $75 \%$. In levels, the respective numbers are $44 \%$ and $89 \%$. Recall that we showed that for a given set of shares $s_{t}^{i}$, s, the cross-sectional dispersion of price dividend ratios increases as $S_{t}$ increases. However, the time variation in $s_{t}^{i}$, possibly also correlated with the market, is an additional source of variation in price dividend ratios. This source of variation in the cross section seems as important in the model as the fluctuations in the stochastic discount factor and hence the failure to capture all the correlation between the price dividend ratio of the market portfolio and the cross sectional standard deviation.

When we concentrate on the first differences the results improve further and the model seems to account for an even larger percentage of the correlation between the first difference of the price dividend ratio of the market portfolio and the first difference of the cross sectional standard deviation of individual price dividend ratios. For instance, these time series have a .75 correlation coefficient in the data whereas the simulations of the model produce a .52 correlation coefficient. The numbers when the series are in logs are .40 and .27 respectively.

\section{DISCUSSION}

\section{VII.A The cash flow model}

This paper models cash flows in order to obtain formulas for prices. Unlike previous models though, ours is a full general equilibrium model where restrictions are imposed in the cash flow processes to make them consistent with the observed US consumption process. A natural question then is whether ours is a "good" model of cash flows. The answer to this question requires a thorough empirical analysis that goes beyond the objective of this paper and certainly the cash flow model could be relaxed along several dimensions. Still we argue that any sensible model of cash flows will conform to the main characteristics of the model proposed in equation (4).

As Bossaerts and Green (1990) recognized, general equilibrium imposes tight restrictions in the set of processes that generate the dividends, otherwise one may posit a process for a particular source of income that may end up accounting for the whole consumption process or even more than it. This would naturally induce rather counterfactual predictions for prices 
and returns. The share process (4) naturally defines each source as a fraction of the overall consumption in a way that prevents any such source from completely dominating consumption. Some of these sources may appear to have trends in sample as they converge to their natural long run level, $\bar{s}^{i}$.

If the share technology is a sensible modeling device, one is left to argue the specific functional form of both the drift and diffusion parts. As for the volatility, what is critical is that the exposure of the share to additional shocks "dies" in the boundary in order to keep it confined in the unit simplex. The behavior of the volatility when the share is far from these boundaries may be important to address some empirical regularities and here we accepted this particular functional form for its convenient tractability.

As for the drift component, a perhaps strong assumption is that we implicitly assume that the investors know the long run contribution of any sector to the overall consumption. It can be shown that the linearity of the model can be used to extend the results here reported to the case where agents use cash flow shocks to learn about $\bar{s}^{i}$. This extension, that we intend to explore in future research, has important implications for the pricing equations. To sketch briefly the intuition behind these results, it is useful to concentrate in the case of Model A in Section II.B.1 . As we saw there, a positive dividend shock that places the share $s_{t}^{i}$ above its long run contribution necessarily results in a decrease in the price dividend ratio. That is, when dividends go up, prices go up as well but by less than the dividend amount (see equation (15)). The reason is that, a positive dividend shock signals weak dividend growth as the share will mean revert to its long run level. Instead, if agents update on $\bar{s}^{i}$, a positive dividend shock yields an upward revision on this estimate, which can, in turn, result in an increase in the price dividend ratio. Essentially learning about $\bar{s}^{i}$ induces additional sources of variation in the prices and returns of individual securities.

\section{VII.B Using price data to learn about the parameters of the cash flow model}

So far we have not used of information other than cash flow data in order to avoid contaminating our assessment of whether the model can account for the empirical regularities of the cross section. Of course, if the purpose is to estimate the parameters of the cash flow model, use of asset pricing data can lead to better estimates of these fundamental parameters. In particular, estimation of the long run shares $\bar{s}^{i}$ can be much improved by the inclusion of financial data for prices contain market expectations about the long run contribution of a 
particular sector to the overall level of consumption. ${ }^{23}$

Although a full fledged estimation of the model using prices and returns deserves a thorough examination, we can compute the level of $\bar{s}^{i}$ that makes the average price-consumption ratio in the data equal to the average fitted price-consumption ratio. We keep all the other parameters, including the shares' speed of mean reversion $\kappa$, as in Table II. The result of this simple exercise is contained in Figure 12. The figure shows that in order to match the average price-consumption ratio, the average long-term share of Petroleum, Construction, Primary Metals, Transportation Eq. and Utilities should be lower than the average historical level, while the ones of Machinery, Electrical Eq., Manufacturing, Dept. Stores and Retail should be higher. In other words, since we can interpret the average $\bar{s}^{i}$ as the expected long-term shares from investors' perspective, the first set of industries are expected to decline in relative size compared to their historical averages, while the second set of industry is expected to increase in relative size.

\section{VII.C The nature of the approximation for prices}

As already mentioned, we can only offer approximate formulas for the general case. To understand the nature of the approximation recall that, in order to obtain a closed form solution for prices, the integral in expression (10) needs to be solved. The heart of the technical problem is then the characterization of the process $q_{t}^{i}=s_{t}^{i} Y_{t}$. By Ito's Lemma, the drift of the stochastic process for $q_{t}^{i}$ includes the term

$$
\left(Y_{t}-\lambda\right) s_{t}^{i} \sum_{j=1}^{n} s_{t}^{j} \theta^{j},
$$

which is quadratic in the shares. However, since we normalized the $\theta^{i}$ 's to have a null unconditional value, $\sum_{j=1}^{n} \bar{s}^{j} \theta^{j}=0$, we can approximate the process for $q_{t}^{i}$ with the one for a stochastic variable $\widehat{q}_{t}^{i}$ defined as

$$
d \widehat{q}_{t}^{i}=d q_{t}^{i}-\left(Y_{t}-\lambda\right) s_{t}^{i} \sum_{j=1}^{n} s_{t}^{j} \theta^{j} d t .
$$

For Model A and B the approximation is exact, as in the former case $Y_{t}=\lambda=1$ and in the

\footnotetext{
${ }^{23}$ Clearly, if we use cross sectional price information to estimate $\bar{s}^{i}$, the fitted price consumption series would be centered around the realized one, and this logically improves the appearance of the plots in Figures 8 and 9. In order to save in space we omit these plots.
} 
latter case $\theta^{i}=0$ for all $i=1, \ldots, n$. For the general case, it turns out that

$$
\left(Y_{t}-\lambda\right) s_{t}^{i} \sum_{j=1}^{n} s_{t}^{j} \theta^{j} d t
$$

is extremely small, leading to an approximation error of less than $0.1 \%$ in prices. Indeed, one can expect the approximation error to be relatively small so long the variance of $\sum_{j=1}^{n} s_{t}^{j} \theta^{j}$ is small. We conjecture that this is always the case when $n$ is sufficiently large. Certainly the volatility of this quantity in our data set, with 20 industry portfolios, is puny and has no pricing impact.

\section{VII.D Comparison with Campbell and Cochrane (1999)}

Our model differs from that of Campbell and Cochrane (1999) in modeling $Y_{t}$ rather than the surplus consumption ratio. A simple application of Ito's Lemma though allows us to compute the differential equation governing the law of motion of $s_{t}=\log \left(S_{t}\right)=-\log \left(Y_{t}\right)$ :

$$
d s_{t}=\left(k\left(1-e^{s_{t}-\bar{s}}\right)+\frac{1}{2} \widehat{\lambda}\left(s_{t}\right)^{2}\right) d t+\widehat{\lambda}\left(s_{t}\right)\left(\frac{d C_{t}}{C_{t}}-\mu_{c}\right)
$$

where

$$
\widehat{\lambda}\left(s_{t}\right)=\alpha\left(1-\lambda e^{s_{t}}\right) \sigma_{c, 1} .
$$

Expression (34) should be compared with equation (3) in Campbell and Cochrane (1999). Besides the Ito term in the drift rate, the model is qualitatively very similar to Campbell and Cochrane (1999): $s_{t}$ follows a mean reverting process with a decreasing volatility in $s_{t}$, as

regulated by a function $\widehat{\lambda}\left(s_{t}\right)$. Differences arise simply to the different functional forms of the drift and volatility.

Campbell and Cochrane (1999) choose the functional form of the sensitivity function, the sensitivity of $s_{t}$ with respect to shocks to consumption growth, to guarantee that the risk free rate remains constant. It is useful then to compare our set up to that of Campbell and Cochrane (1999) in light of equation (25). As it is well known, a drawback of habit persistence models is that the dependence of the risk free rate on $S_{t}$ may result in rates that are too volatile when compared with data. It is for this reason that Campbell and Cochrane (1999) judiciously choose functional forms for the dynamics of the surplus consumption ratio that guarantee stable interest rates. Equation (25) shows the parametric restrictions that are needed in order to yield a constant risk free rate:

$$
\alpha=\frac{k}{\lambda \sigma_{c} \sigma_{c}^{\prime} \bar{S}},
$$


which is, essentially, the modeling choice made by Campbell and Cochrane (1999). We have not imposed condition (35). ${ }^{24}$ There were two reasons to do this. First, we wanted our model to deliver implications for both the risk free rate and the stock market to provide as complete a picture as possible of financial markets. The second reason is linked to the lower risk-aversion coefficient that we assume compared to Campbell and Cochrane (1999). If we were to impose (35), stabilizing the interest rate would result in a very highly volatile habit level, which is difficult to reconcile with its intuition of being a smoothed average of past consumption levels.

\section{CONCLUSIONS}

If one is interested in prices modeling cash flows is unavoidable. In this paper we advance a model of cash flows that we combine with a stochastic discount factor to solve for prices within a general equilibrium consumption based asset pricing model. Prices in our model are given by a term that is linear in what dividends should be, given the current consumption level, a stochastic trend, and a second term that is the deviation of the current level of dividends with respect to their stochastic trend. The coefficient in each of these two terms depend on the variable that summarizes the aggregate state of the economy, the surplus consumption ratio. This representation sheds light on several issues.

Shocks to cash flows have very different effects on prices depending on where they occur over the business cycle. In particular, when cash flow betas are identical across assets, cash flow shocks have a large impact on prices during business cycle peaks and a smaller effect in troughs. Conversely, the business cycle drives the cross section of price dividend ratios in predictable ways. Once again, if assets have identical cash flow betas, the dispersion of individual price dividend ratios increases over business cycle peaks. This result survives when we allow for a general covariance structure between dividend growth across different assets and consumption growth, as model simulations show. This matches the empirical regularity uncovered in this paper, namely, that the cross sectional standard deviation of price dividend ratio tracks the level of the price dividend ratio of the market portfolio.

\footnotetext{
${ }^{24}$ Neither do Campbell and Cochrane (1999) in the working paper version of their paper. See also their discussion in pages 214-216 of the published version. Notice also that the source of variation in the risk free rate is different from the one recently explored by Wachter (2001), where the real rate changes due to changes in the drift rate of consumption. In our paper, as in Campbell and Cochrane (1999), consumption growth is an i.i.d. process.
} 
The model matched the cross sectional dispersion in the variance decomposition across industries as well as the observed history of prices across our set of industry portfolios. In particular, the model links secular trends in prices to the lower share that these assets contribute to overall consumption but shows that business cycle considerations need to be included in order to fully account for the history of price levels. ${ }^{25}$

There is an important aspect of the ideas advanced in this paper that we intend to tackle in future research. Our set up is one that allows for a full specification of the conditional moments of financial variables, whether price dividend ratios or returns, and, consequently, we can identify and study these conditional moments through the lenses of the model. To motivate this exercise, it is useful to return to Figure 2. In addition to the level of prices, the main motivation to include it early in the paper, the plot shows a rather intriguing difference between Paper and Railroads. The time series of these two industries are almost the mirror image of each other. Railroads tracks the price consumption ratio of the market portfolio until 1960 when they uncouple and move in opposite direction. Up to 1960 then Railroads seems driven mostly by a common aggregate factor whereas afterwards it comoves less with the market, to the point where it does not even react significantly to the correction in the mid 1970s. Paper is exactly the opposite. It seems to be largely disconnected of the aggregate price consumption ratio in the early part of the sample and it is only after 1960 that it shows the same business cycle patterns that characterize the market, though Paper did not experience the sudden and extraordinary rise of aggregate prices in the second half of the 90s. Indeed, the variance decomposition looks rather different across industries when the sample is split evenly. In the 1927-1962 sample, the variance of the price dividend ratio for Railroads is only driven by shocks to expected returns whereas in the 1963-2001 sample is the exact opposite. For Paper shocks to expected returns is a much large component of the variance of the price dividend ratio throughout.

It seems then that the weight of each component on the variance of the price dividend ratio is changing over time. We believe that, in general, looking at these and other conditional moments can give additional insights into asset prices and, perhaps more importantly, into the elusive connection between finance and macroeconomics.

\footnotetext{
${ }^{25}$ Clearly, though our model is supposed to explain prices at any frequency, it cannot reproduce the behavior of prices at very high frequencies, and ingredients others than the ones brought to bear here need to be introduced to account for them.
} 


\section{REFERENCES}

Abel, Andrew B. (1990) "Asset Prices Under Habit Formation and Catching Up with the Jones," American Economic Review, 80, 38-42.

Bakshi, Gurdip S. and Zhiwu Chen (1996), "The Spirit of Capitalism and Stock Prices," American Economic Review, 86 (1), 133-157.

Boldrin, Michele, Lawrence J. Christiano, and Jonas D. Fisher, (2001), "Habit Persistence, Asset Returns, and the Business Cycle, American Economic Review, 91 (1), March, 149-166.

Bossaerts, Peter and Richard C. Green (1989), "A General Equilibrium Model of Changing Risk Premia: Theory and Tests," Review of Financial Studies, vol. 2 (4), 467-493.

Boudouck, Jacob, Matthew Richardson, and Robert F. Whitelaw, (1994) "Industry Returns and the Fisher Effect," Journal of Finance, 49, 5, 1595-1615.

Brock, William A. (1982), "Asset Prices in a Production Economy," in Jon J. McCall ed.: The Economics of Information and Uncertainty. The University of Chicago Press, Chicago, IL.

Campbell, John Y. (2001), "Consumption-based Asset Pricing," forthcoming in Handbook of the Economics and Finance, by George Constantinides, Milton Harris and Rene Stulz (Eds.), North Holland, Amsterdam.

Campbell, John Y. and N. Gregory Mankiw (1990), "Permanent Income, Current Income, and Consumption," Journal of Business $\& 3$ Economic Statistics, July, 8 (3), 265-279.

Campbell, John Y. and John H. Cochrane (1999), "By Force of Habit: A Consumption Based Explanation of Aggregate Stock Market Behavior," Journal of Political Economy, 107, 2, 205251.

Campbell, John Y., Martin Lettau, Burton Malkiel and Yexiao Xu (2001), "Have Individual Stocks Become More Volatile? An Empirical Exploration of Idiosyncratic Risk." Journal of Finance, 56, 1, 1-43.

Campbell, John Y., Andrew W. Lo, and A. Craig MacKinlay (1997), The Econometrics of Financial Markets. Princeton University Press, Princeton, New Jersey. 
Carroll, Christopher D., Jody Overland, and David N. Weil, (2000), "Saving and Growth with Habit Formation," American Economic Review, 90 (3), June, 341-355.

Chapman, David A. (1998), "Habit Formation and Aggregate Consumption," Econometrica, 66 (5), September, 1223-1230.

Cochrane, John H. (1991), "Explaining the Variance of Price Dividend Ratios," Review of Financial Studies, 5, 243-280.

Cochrane, John H. (2000), Asset Pricing, Princeton University Press, Princeton, NJ.

Constantinides, George (1990), "Habit Formation: A Resolution of the Equity Premium Puzzle," Journal of Political Economy, 98, 519-543.

Daniel, Kent and David Marshall (1997), "The Equity Premium Puzzle and the Risk-Free Rate Puzzle at Long Horizons," Macroeconomic Dynamics, 1 (2), 452-484.

Deaton, Angus and John Muellbauer (1980), "Economics and Consumer Behavior. New York: Cambridge University Press.

Detemple, Jerome B. and Fernando Zapatero, (1991), "Asset Prices in an Exchange Economy with Habit Formation," Econometrica, Vol. 59, pp. 1633-1657.

Duesenberry, James S. (1949), Income, Saving, and the Theory of Consumer Behavior. New York: Oxford University Press.

Dynan, Karen E. (2000), "Habit Formation in Consumer Preferences: Evidence from Panel Data," American Economic Review, 90 (3), June, 391-406.

Ferson, Wayne and George Constantinides, (1991), "Habit Persistence and Durability in Aggregate Consumption: Empirical Tests," Journal of Financial Economics, 29, 199-240.

Grinblatt, Mark and Toby Moskowitz (1999), "Do Industries Explain Momentum," Journal of Finance, 54, 1249-1290.

Heaton, John C. (1993), "The Interaction Between Time-Nonseparable Preferences and Time Aggregation," Econometrica 63, 681-717. 
Heien, Dale and Cathy Durham (1991), "A Test of Habit Formation Hypothesis Using Household Data," The Review of Economics and Statistics, 74 (2), May, 189-199.

Lettau, Martin and Harlad Uhlig (2000), "Can Habit Formation be Reconciled with Business Cycle Facts," Review of Economic Dynamics, 3 (1), 79-99.

Li, Yuming (2001), "Expected Returns and Habit Persistence," The Review of Financial Studies, 14, 861-899.

Lucas, Robert E. (1978), "Asset Prices in an Exchange Economy," Econometrica 46, 1429-1445.

Menzli, Lior, Tano Santos, and Pietro Veronesi (2002), "Habit Formation and the Cross Section of Stock Returns," manuscript, Graduate School of Business, The University of Chicago.

Moskowitz, Tobias and Mark Grinblatt (1999), "Do Industries Explain Momentum?" Journal of Finance.

Santos, Tano and Pietro Veronesi (2001), "Labor Income and Predictable Stock Returns," manuscript, Graduate School of Business, The University of Chicago.

Sundaresan, Suresh (1989), "Intertemporal Dependent Preferences and the Volatility of Consumption and Wealth," The Review of Financial Studies, 2, 73-88.

Vuolteenaho, Tuomo (2002), "What Drives Firm-Level Stock Returns?" Journal of Finance, 57, 233-264.

Wachter, Jessica (2000), Habit Formation and the Cross-Section of Asset Returns, Unpublised Doctoral Dissertation, Ch. 4, Department of Economics, Harvard University.

Wachter, Jessica (2001), "Habit Formation and Returns on Bonds and Stocks," manuscript, Stern School of Business, New York University. 


\section{APPENDIX}

\section{(A) The stationary density of $Y_{t}$}

The stationary density for the process $Y$ in equation (7) depends only on three parameters, $\bar{Y}, \lambda$ and $b=k /\left(\alpha^{2} \sigma_{c} \sigma_{c}^{\prime}\right)$ and it is given by

$$
\psi(Y)=\frac{e^{-2 b \frac{\bar{Y}-\lambda}{(Y-\lambda)} \times(Y-\lambda)^{-2 b-2}}}{\int_{\lambda}^{\infty} e^{-2 b \frac{\bar{Y}-\lambda}{(y-\lambda)}} \times(y-\lambda)^{-2 b-2} d y} .
$$

We use equation (36) to compute the unconditional moments of aggregate variables. We match these unconditional moments to their sample counterparts in the calibration described in Section V. Specifically, we choose the parameters $\bar{Y}, \lambda, k$ and $\alpha$ to match the following moments: ${ }^{26}$

$$
\begin{aligned}
E\left[d R_{t}^{T W}\right] & =\int_{\lambda}^{\infty} \mu^{T W}(Y) \psi(Y) d Y=\text { Data } \\
E\left[r^{f}(Y)\right] & =\int_{\lambda}^{\infty} r(Y) \psi(Y) d Y=\text { Data } \\
E\left[d r^{f}(Y)^{2}\right] & =\int_{\lambda}^{\infty} \sigma_{r}^{2}(Y) \psi(Y) d Y=\text { Data } \\
E\left[\frac{P}{C}(Y)\right] & =\int_{\lambda}^{\infty} \frac{P}{C}(Y) \psi(Y) d Y=\text { Data } \\
\frac{E\left[d R_{t}^{T W}\right]}{\sqrt{E\left[\left(d R_{t}^{T W}\right)^{2}\right]}} & =\frac{\int_{\lambda}^{\infty} \mu^{T W}(Y) \psi(Y) d Y}{\sqrt{\int_{\lambda}^{\infty}\left\|\sigma^{T W}(Y)\right\|^{2} \psi(Y) d Y}}=\text { Data. }
\end{aligned}
$$

\section{(B) The Share Process}

We estimate the parameters in two steps: First, we estimate the parameters $\bar{s}^{i}$ and $\kappa$ by applying standard GMM arguments to the discretized Euler equation

$$
s_{t+\Delta}^{i}=\bar{s}^{i} \kappa+(1-\kappa) s_{t}^{i}+\varepsilon_{t+\Delta}^{i}
$$

where $\varepsilon_{t+\Delta}^{i}$ such that

$$
\begin{aligned}
\mathbf{E}\left[\varepsilon_{t+\Delta}^{i} \mid \varepsilon_{t}, \varepsilon_{t-\Delta}, \ldots, \mathbf{s}_{t}, \mathbf{s}_{t-\Delta}\right] & =0 \\
\mathbf{E}\left[\left(\varepsilon_{t+\Delta}^{i}\right)^{2} \mid \varepsilon_{t}, \varepsilon_{t-\Delta}, \ldots, \mathbf{s}_{t}, \mathbf{s}_{t-\Delta}\right] & =\sigma^{i}\left(\mathbf{s}_{t}\right) \sigma^{i}\left(\mathbf{s}_{t}\right)^{\prime}
\end{aligned}
$$

\footnotetext{
${ }^{26}$ The last equation matches the Sharpe ratio, computed in the data as the ratio of mean stock returns over its standard deviation. Although the model has a closed form expression for the Sharpe ratio (see equation (28)), matching its unconditional average to its sample counterpart would not take into account a Jensens' inequality term. The appropriate procedure is to compute the model-implied ratio of the unconditional mean return to the unconditional mean volatility, and match that to its sample counterpart.
} 
for some function $\sigma^{i}\left(\mathbf{s}_{t}\right)$. Defining $\mathbf{e}_{t+\Delta}=\mathbf{s}_{t+\Delta}-\overline{\mathbf{s}} k-(1-k) \mathbf{s}_{t}$, we impose the moment conditions

$$
E\left[\mathbf{e}_{t+\Delta}\right]=0 \text { and } E\left[\mathbf{e}_{t+\Delta} \odot \mathbf{s}_{t}\right]=0
$$

Since the model effectively predicts a stochastic volatility for the errors $\mathbf{e}_{t+\Delta}$, we estimate the parameters by efficient GMM with Newey-West spectral density matrix

$$
\mathbf{S}_{T}=\boldsymbol{\Gamma}_{0}+\sum_{v=1}^{q}(1-(v /(q+1)))\left(\boldsymbol{\Gamma}_{v}+\boldsymbol{\Gamma}_{v}^{\prime}\right)
$$

where $\boldsymbol{\Gamma}_{v}=E\left[\mathbf{u}_{t} \mathbf{u}_{t-v}\right]$ is the $v$-lag autocovariance matrix of (de-meaned) errors $u_{t}$. The number of lags $q$ was chosen to equal four (quarters).

Next, we turn to estimate the vectors $\mathbf{v}^{i}$ 's. Notice that the diffusion part of the process $\log \left(s_{t}^{i}\right)$ is given by $\sigma_{\log }^{i}=\mathbf{v}^{i}-\sum_{j=1}^{n} s_{t}^{j} \mathbf{v}^{j}$. We rely on an approximation of this volatility function. Monte Carlo simulations show that the methodology is accurate. If we assume that the $\sum_{j=1}^{n} s_{t}^{j} \mathbf{v}^{j}$ component of $\sigma_{\log }^{i}$ does not move by a good deal over the sample period, then we can assume it constant and approximately equal to $\sum_{j=1}^{n} \bar{s}^{j} \mathbf{v}^{j}$. Hence the diffusion process of the $\log$-share would be $\sigma_{\log }^{i} \approx \mathbf{v}^{i}-\sum_{j=1}^{n} \mathbf{v}^{j} \bar{s}^{j}$. In addition, because shares sum up to one, without loss of generality we can assume $\mathbf{v}^{n}=0$. Hence, define the vector $\mathbf{y}_{t}=$ $\left[\Delta \log \left(C_{t}\right), \Delta \log \left(s_{t}^{1}\right), \ldots, \Delta \log \left(s_{t}^{n-1}\right)\right]^{\prime}$ and compute

$$
\left(\begin{array}{c}
\sigma_{c} \\
\sigma_{\log }^{1} \\
\vdots \\
\sigma_{\log }^{n-1}
\end{array}\right)=\operatorname{Chol}\left(\frac{1}{T} \sum_{t=1}^{T} \mathbf{y}_{t} \mathbf{y}_{t}^{\prime}\right)
$$

Hence, we can impose

$$
\begin{aligned}
\sigma_{\log } & \equiv\left(\begin{array}{c}
\sigma_{\log }^{1} \\
\vdots \\
\sigma_{\log }^{n-1}
\end{array}\right)=\left(\begin{array}{c}
\mathbf{v}^{1} \\
\vdots \\
\mathbf{v}^{n-1}
\end{array}\right)-\sum_{j=1}^{n-1} \mathbf{v}^{j} \bar{s}^{j} \\
& =\mathbf{v}^{(-n)}-\mathbf{1}_{n-1} \overline{\mathbf{s}}^{(-n) \prime} \mathbf{v} \\
& =\left(\mathbf{I}_{n-1}-\mathbf{1}_{n-1} \overline{\mathbf{s}}^{(-n) \prime}\right) \mathbf{v}^{(-n)}
\end{aligned}
$$

which finally implies

$$
\mathbf{v}^{(-n)}=\left(\mathbf{I}-\mathbf{1}_{n-1} \overline{\mathbf{s}}^{(-n)^{\prime}}\right)^{-1} \sigma_{\log }^{(-n)}
$$


We use this formula to calibrate the model to the share process.

Once we estimate the $\mathbf{v}^{i}$, s, we can renormalize them in order to make $\sum_{i=1}^{n} \bar{s}^{i} \mathbf{v}^{i}=0$ and so impose (5).

\section{(C) Derivation of the pricing formulas.}

In this appendix we only provide the explicit steps about the approximate formula (20). Special cases, and exact, of the following derivation are the pricing functions in Proposition 1 - 3, which can be derived directly following the steps in the text.

Define for convenience $\mathbf{v}_{Y}=-\alpha \sigma_{c}$ so that the Habit process can be rewritten as

$$
d Y_{t}=k\left(\bar{Y}-Y_{t}\right) d t+\left(Y_{t}-\lambda\right) \mathbf{v}_{Y} d \mathbf{B}_{t}
$$

Given the form of the utility function, as before, we obtain immediately the price of the stock:

$$
P_{t}^{i}=\left(C_{t}-X_{t}\right) E_{t}\left[\int_{0}^{\infty} e^{-\phi \tau} q_{t+\tau}^{i} d \tau\right]
$$

where $q_{t}^{i}=s_{t}^{i} Y_{t}$. Notice first that since $0<q_{t}^{i}<Y_{t}$ for all $i$, if $E_{t}\left[\int_{0}^{\infty} e^{-\phi \tau} Y_{t+\tau} d \tau\right]<$ $\infty$ we can invoke Fubini's theorem and invert the order of intergration, to obtain $P_{t}^{i}=$ $\left(C_{t}-X_{t}\right) \int_{0}^{\infty} e^{-\phi \tau} E_{t}\left[q_{t+\tau}^{i}\right] d \tau$. We assume that the parametric restrictions on $\phi, k$ and $\lambda$ ensure $E_{t}\left[\int_{0}^{\infty} e^{-\phi \tau} Y_{t+\tau} d \tau\right]<\infty$ (see Karatzas and Shreve (1991, page360-361) for conditions). Using Ito's lemma, we can write

$$
\begin{aligned}
d q_{t}^{i}= & \left\{\kappa\left(Y_{t} \bar{s}^{i}-q_{t}^{i}\right)+k\left(s_{t}^{i} \bar{Y}-q_{t}^{i}\right)-\alpha\left(Y_{t}-\lambda\right) s_{t}^{i} \theta^{i}+\left(Y_{t}-\lambda\right) s_{t}^{i} \sum_{j=1}^{n} s_{t}^{j} \theta^{j}\right\} d t \\
& +\left\{q_{t}^{i}\left(\mathbf{v}_{i}-\sum_{j=1}^{n} s_{t}^{j} \mathbf{v}_{j}\right)+s_{t}^{i}\left(Y_{t}-\lambda\right) \mathbf{v}_{Y}\right\} d \mathbf{B}_{t}
\end{aligned}
$$

We now use (5) and approximate the term $\sum_{j=1}^{n} s_{t}^{j} \theta_{j}$ by $E\left[\sum_{j=1}^{n} s_{t}^{j} \theta_{j}\right]=\sum_{j=1}^{n} \bar{s}^{j} \theta_{j}=0$. Given $n$ large, if the cross-sectional dispersion of $\theta^{i}$ 's is not too large, the time-variation of $\sum_{j=1}^{n} s_{t}^{j} \theta_{j}$ is actually small. Our simulations show that the approximate price is within $0.1 \%$ from its actual price. Thus, pricing will be based on the approximate process:

$d q_{t}^{i}=\left\{\kappa \bar{s}^{i} Y_{t}+\left(k \bar{Y}+\alpha \lambda \theta_{i}\right) s_{t}^{i}-\left(\kappa+k+\alpha \theta_{i}\right) q_{t}^{i}\right\} d t+\left\{q_{t}^{i}\left(\mathbf{v}_{i}-\sum_{j=1}^{n} s_{t}^{j} \mathbf{v}_{j}\right)+s_{t}^{i}\left(Y_{t}-\lambda\right) \mathbf{v}_{Y}\right\} d \mathbf{B}_{t}$ 
Define $\mathbf{Z}_{t}^{i}=\left(Y_{t}, q_{t}^{i}, s_{t}^{i}\right)^{\prime}$. This evolves according to the system $d \mathbf{Z}_{t}=\mathbf{A}_{0}+\mathbf{A}_{1} \mathbf{Z}_{t}+\boldsymbol{\Sigma}\left(Y_{t}, \mathbf{s}_{t}\right) d \mathbf{B}_{t}$ where $\mathbf{A}_{0}=\left(k \bar{Y}, 0, \kappa \bar{s}^{i}\right)^{\prime}$,

$$
\mathbf{A}_{1}=\left(\begin{array}{lll}
-k & 0 & 0 \\
\kappa \bar{s}^{i} & -\left(\kappa+k+\alpha \theta_{i}\right) & k \bar{Y}+\lambda \alpha \theta_{i} \\
0 & 0 & -\kappa
\end{array}\right)
$$

and $\boldsymbol{\Sigma}\left(Y_{t}, \mathbf{s}_{t}\right)$ is a $3 \times n$ appropriate matrix. Since it is linear, and since $\mathbf{A}_{1}$ admits 3 distinct real eigenvalues, we have

$$
E_{t}\left[\mathbf{Z}_{t+\tau}\right]=\mathbf{\Psi}(\tau) \mathbf{Z}_{t}+\int_{t}^{\tau} \boldsymbol{\Psi}(\tau-s) \mathbf{A}_{0} d s
$$

with $\boldsymbol{\Psi}(\tau)=\mathbf{U} \exp (\boldsymbol{\Lambda} \cdot \tau) \mathbf{U}^{-1}$, where $\boldsymbol{\Lambda}$ is the diagonal matrix with eigenvalues of $\mathbf{A}_{1}^{i}$ and $\mathbf{U}$ is the matrix of associated eigenvectors. Since direct computation show that

$$
\boldsymbol{\Lambda}=\left(\begin{array}{lll}
-\kappa & 0 & 0 \\
0 & -k & 0 \\
0 & 0 & -\left(\kappa+k+\alpha \theta_{i}\right)
\end{array}\right) \text { and } \mathbf{U}=\left(\begin{array}{lll}
0 & \frac{\kappa+\alpha \theta_{i}}{\kappa \bar{s}^{i}} & 0 \\
\frac{k \bar{Y}+\lambda \alpha \theta_{i}}{k+\alpha \theta_{i}} & 1 & 1 \\
1 & 0 & 0
\end{array}\right)
$$

we have the solution

$$
\boldsymbol{\Psi}(\tau)=\left(\begin{array}{ccc}
e^{-k \tau} & 0 & 0 \\
\frac{\kappa \bar{s}_{i}}{\kappa+\alpha \theta_{i}}\left(e^{-k \tau}-e^{-\left(k+\kappa+\alpha \theta_{i}\right) \tau}\right) & e^{-\left(k+\kappa+\alpha \theta_{i}\right) \tau} & \frac{\left(k \bar{Y}+\lambda \alpha \theta_{i}\right)}{k+\alpha \theta_{i}}\left(e^{-\kappa \tau}-e^{-\left(k+\kappa+\alpha \theta_{i}\right) \tau}\right) \\
0 & 0 & e^{-\kappa \tau}
\end{array}\right)
$$

Therefore, in order to obtain the price, we need to compute

$$
E_{t}\left[q_{t+\tau}^{i}\right]=e_{2} E_{t}\left[Z_{t+\tau}\right]=e_{2} \boldsymbol{\Psi}(\tau) \mathbf{Z}_{t}+\int_{0}^{\tau} e_{2} \mathbf{\Psi}(\tau-s) \mathbf{A}_{0} d s
$$

where $e_{2}=(0,1,0)$. First, we have

$$
\begin{aligned}
e_{2} \Psi(\tau) \mathbf{Z}_{t}= & \frac{\kappa \bar{s}_{i}}{\kappa+\alpha \theta_{i}}\left(e^{-k \tau}-e^{-\left(k+\kappa+\alpha \theta_{i}\right) \tau}\right) Y_{t}+e^{-\left(k+\kappa+\alpha \theta_{i}\right) \tau} q_{t}^{i} \\
& +\frac{\left(k \bar{Y}+\lambda \alpha \theta_{i}\right)}{k+\alpha \theta_{i}}\left(e^{-\kappa \tau}-e^{-\left(k+\kappa+\alpha \theta_{i}\right) \tau}\right) s_{t}^{i}
\end{aligned}
$$

Similarly

$$
\begin{aligned}
e_{2} \mathbf{\Psi}(\tau-s) \mathbf{A}_{0}= & \frac{\kappa \bar{s}_{i}}{\kappa+\alpha \theta_{i}}\left(e^{-k(\tau-s)}-e^{-\left(k+\kappa+\alpha \theta_{i}\right)(\tau-s)}\right) k \bar{Y} \\
& +\frac{\left(k \bar{Y}+\lambda \alpha \theta_{i}\right)}{k+\alpha \theta_{i}}\left(e^{-\kappa(\tau-s)}-e^{-\left(k+\kappa+\alpha \theta_{i}\right)(\tau-s)}\right) \kappa \bar{s}^{i}
\end{aligned}
$$


Since

$$
\int_{0}^{\tau} e^{-a(\tau-s)} d s=e^{-a \tau} \int_{0}^{\tau} e^{a s} d s=e^{-a \tau} \frac{e^{a \tau}-1}{a}=\frac{1-e^{-a \tau}}{a}
$$

we obtain

$$
\begin{aligned}
\int_{0}^{\tau} e_{2} \boldsymbol{\Psi}(\tau-s) \mathbf{A}_{0} d s= & \frac{\kappa \bar{s}_{i} k \bar{Y}}{\kappa+\alpha \theta_{i}}\left(\frac{1-e^{-k \tau}}{k}-\frac{1-e^{-\left(k+\kappa+\alpha \theta_{i}\right) \tau}}{\left(k+\kappa+\alpha \theta_{i}\right)}\right) \\
& +\frac{\left(k \bar{Y}+\lambda \alpha \theta_{i}\right) \kappa \bar{s}^{i}}{k+\alpha \theta_{i}}\left(\frac{1-e^{-\kappa \tau}}{\kappa}-\frac{1-e^{-\left(k+\kappa+\alpha \theta_{i}\right) \tau}}{\left(k+\kappa+\alpha \theta_{i}\right)}\right)
\end{aligned}
$$

We then have (approximately)

$$
E_{t}\left[\int_{t}^{\infty} e^{-\phi(\tau-t)} q_{\tau}^{i} d \tau\right]=\int_{0}^{\infty} e^{-\phi \tau} e_{2} \boldsymbol{\Psi}(\tau) \mathbf{Z}_{t} d \tau+\int_{0}^{\infty} e^{-\phi \tau} \int_{0}^{\tau} e_{2} \boldsymbol{\Psi}(\tau-s) \mathbf{A}_{0} d s d \tau
$$

Recalling that for any constant $a>0, \int_{0}^{\infty} e^{-a \tau} d \tau=\left[\frac{e^{-a \tau}}{-a}\right]_{\tau=0}^{\tau=\infty}=\frac{1}{a}$, using the results above, we obtain (after tedious algebra):

$$
\int_{0}^{\infty} e^{-\phi \tau} \int_{0}^{\tau} e_{2} \boldsymbol{\Psi}(\tau-s) \mathbf{A}_{0} d s d \tau=\frac{\kappa \bar{s}_{i} k \bar{Y}}{\phi(\phi+k)\left(\phi+k+\kappa+\alpha \theta_{i}\right)}+\frac{\left(k \bar{Y}+\lambda \alpha \theta_{i}\right) \kappa \bar{s}^{i}}{\phi(\phi+\kappa)\left(\phi+k+\kappa+\alpha \theta_{i}\right)}
$$

and, similarly

$$
\begin{aligned}
\int_{0}^{\infty} e^{-\phi \tau} e_{2} \mathbf{\Psi}(\tau) \mathbf{Z}_{t} d \tau= & \frac{\kappa \bar{s}_{i}}{(\phi+k)\left(\phi+k+\kappa+\alpha \theta_{i}\right)} Y_{t}+\frac{1}{\left(\phi+k+\kappa+\alpha \theta_{i}\right)} q_{t}^{i} \\
& +\frac{\left(k \bar{Y}+\lambda \alpha \theta_{i}\right)}{(\phi+\kappa)\left(\phi+k+\kappa+\alpha \theta_{i}\right)} s_{t}^{i}
\end{aligned}
$$

Thus,

$$
\begin{aligned}
E_{t}\left[\int_{t}^{\infty} e^{-\phi(\tau-t)} q_{\tau}^{i} d \tau\right] & =\frac{\kappa \bar{s}_{i}}{(\phi+k)\left(\phi+k+\kappa+\alpha \theta_{i}\right)} Y_{t}+\frac{1}{\left(\phi+k+\kappa+\alpha \theta_{i}\right)} q_{t}^{i} \\
& +\frac{\left(k \bar{Y}+\lambda \alpha \theta_{i}\right)}{(\phi+\kappa)\left(\phi+k+\kappa+\alpha \theta_{i}\right)} s_{t}^{i} \\
& +\frac{\kappa \bar{s}_{i} k \bar{Y}}{\phi(\phi+k)\left(\phi+k+\kappa+\alpha \theta_{i}\right)}+\frac{\left(k \bar{Y}+\lambda \alpha \theta_{i}\right) \kappa \bar{s}^{i}}{\phi(\phi+\kappa)\left(\phi+k+\kappa+\alpha \theta_{i}\right)}
\end{aligned}
$$

Finally, using $\left(C_{t}-X_{t}\right)=C_{t} / Y_{t}$, we find

$$
\begin{aligned}
\frac{P_{t}^{i}}{C_{t}}= & \frac{1}{Y_{t}} E_{t}\left[\int_{t}^{\infty} e^{-\phi(\tau-t)} q_{\tau}^{i} d \tau\right] \\
= & \frac{\kappa \bar{s}_{i}}{(\phi+k)\left(\phi+k+\kappa+\alpha \theta_{i}\right)}+\frac{1}{\left(\phi+k+\kappa+\alpha \theta_{i}\right)} s_{t}^{i}+\frac{\left(k \bar{Y}+\lambda \alpha \theta_{i}\right)}{(\phi+\kappa)\left(\phi+k+\kappa+\alpha \theta_{i}\right)} \frac{s_{t}^{i}}{Y_{t}} \\
& +\frac{\kappa \bar{s}_{i} k \bar{Y}}{\phi(\phi+k)\left(\phi+k+\kappa+\alpha \theta_{i}\right)} \frac{1}{Y_{t}}+\frac{\left(k \bar{Y}+\lambda \alpha \theta_{i}\right) \kappa \bar{s}^{i}}{\phi(\phi+\kappa)\left(\phi+k+\kappa+\alpha \theta_{i}\right)} \frac{1}{Y_{t}}
\end{aligned}
$$


which can be rewritten as

$$
\begin{aligned}
\frac{P_{t}^{i}}{C_{t}}= & \frac{\kappa \bar{s}_{i}}{(\phi+k)\left(\phi+k+\kappa+\alpha \theta_{i}\right)}\left(1+\frac{k \bar{Y}}{\phi} S_{t}\right) \\
& +\frac{1}{\left(\phi+k+\kappa+\alpha \theta_{i}\right)}\left(s_{t}^{i}+\frac{k \bar{Y}+\lambda \alpha \theta_{i}}{\phi+\kappa} S_{t}\left(s_{t}^{i}+\frac{\kappa}{\phi} \bar{s}^{i}\right)\right)
\end{aligned}
$$

and then, upon definition of $\omega^{i}=\alpha \theta^{i} /\left(\phi+k+\kappa+\alpha \theta_{i}\right)$ and some more algebric manipulations, as (20).

Proof of Proposition 4. It is immediate when taking the cross sectional variance in expression (19) and taking the derivative with respect to $S_{t}$.

\section{(D) Derivation of the return formulas.}

To obtain the approximate return formula, an exact one for propositions 5 and 6 , we define $\rho_{t}^{i}=P_{t}^{i} / C_{t}$ which, for notational convenience, is denoted by

$$
\rho_{t}^{i}=a_{0}^{i}+a_{1}^{i} S_{t}+a_{2}^{i} s_{t}^{i}+a_{3}^{i} s_{t}^{i} S_{t}
$$

where

$$
\begin{aligned}
a_{0}^{i} & =\frac{\kappa \bar{s}_{i}}{(\phi+k)\left(\phi+k+\kappa+\alpha \theta_{i}\right)}=\frac{\kappa \bar{s}_{i}}{(\phi+k)} a_{2}^{i} \\
a_{1}^{i} & =\frac{\kappa \bar{s}_{i}}{(\phi+k)\left(\phi+k+\kappa+\alpha \theta_{i}\right)} \frac{\bar{Y} k}{\phi}+\frac{1}{\left(\phi+k+\kappa+\alpha \theta_{i}\right)} \frac{\left(k \bar{Y}+\lambda \alpha \theta_{i}\right)}{(\phi+\kappa)} \frac{\kappa}{\phi} \bar{s}^{i} \\
& =a_{2}^{i} \frac{1}{\phi}\left(\frac{\kappa \bar{s}_{i} \bar{Y} k(\phi+\kappa)+(\phi+k)\left(k \bar{Y}+\lambda \alpha \theta_{i}\right) \kappa \bar{s}^{i}}{(\phi+k)(\phi+\kappa)}\right) \\
& =a_{2}^{i}\left(\frac{\kappa \bar{s}_{i} \bar{Y} k(2 \phi+k+\kappa)+(\phi+k) \lambda \alpha \theta_{i} \kappa \bar{s}^{i}}{\phi(\phi+k)(\phi+\kappa)}\right) \\
a_{2}^{i} & =\frac{1}{\left(\phi+k+\kappa+\alpha \theta_{i}\right)} \\
a_{3}^{i} & =\frac{1}{\left(\phi+k+\kappa+\alpha \theta_{i}\right)} \frac{\left(k \bar{Y}+\lambda \alpha \theta_{i}\right)}{(\phi+\kappa)} \\
& =a_{2}^{i} \frac{\left(k \bar{Y}+\lambda \alpha \theta_{i}\right)}{(\phi+\kappa)}
\end{aligned}
$$

The definition $S_{t}=1 / Y_{t}$ implies

$$
\frac{d S_{t}}{S_{t}}=\left(k\left(1-\frac{S_{t}}{\bar{S}}\right)+\left(1-S_{t} \lambda\right)^{2} \mathbf{v}_{Y} \mathbf{v}_{Y}^{\prime}\right) d t-\left(1-\lambda S_{t}\right) \mathbf{v}_{Y} d \mathbf{B}_{t}
$$

Hence

$$
d \rho_{t}^{i}=a_{1}^{i} d S_{t}+a_{2}^{i} d s_{t}^{i}+a_{3}^{i}\left(S_{t} d s_{t}^{i}+s_{t}^{i} d S_{t}+d s_{t}^{i} d S_{t}\right)
$$




$$
\begin{gathered}
=\quad \mu_{\rho, t}^{i} d t-\left(a_{1}^{i}+a_{3}^{i} s_{t}^{i}\right) S_{t}\left(1-\lambda S_{t}\right) \mathbf{v}_{Y} d \mathbf{B}_{t}+ \\
+\left(a_{2}^{i}+a_{3}^{i} S_{t}\right) s_{t}^{i}\left(\mathbf{v}^{i}-\sum_{j=1}^{n} s_{t}^{j} \mathbf{v}^{j}\right) d \mathbf{B}_{t}
\end{gathered}
$$

where $\mu_{\rho, t}$ contains all the "dt" terms. Therefore

$$
\begin{aligned}
d P_{t}^{i} & =\rho_{t}^{i} d C_{t}+C_{t} d \rho_{t}^{i}+d C_{t} d \rho_{t}^{i} \\
& =P_{t}^{i} \mu_{P, t}^{i} d t+P_{t}^{i} \sigma_{P, t}^{i} d \mathbf{B}_{t}
\end{aligned}
$$

with

$$
\begin{aligned}
\sigma_{P, t}^{i} & =\sigma_{c}-\frac{\left(a_{1}^{i}+a_{3}^{i} s_{t}^{i}\right) S_{t}\left(1-\lambda S_{t}\right)}{a_{0}^{i}+a_{1}^{i} S_{t}+a_{2}^{i} s_{t}^{i}+a_{3}^{i} s_{t}^{i} S_{t}} \mathbf{v}_{Y}+\frac{\left(a_{2}^{i}+a_{3}^{i} S_{t}\right) s_{t}^{i}\left(\mathbf{v}^{i}-\sum_{j=1}^{n} s_{t}^{j} \mathbf{v}^{j}\right)}{a_{0}^{i}+a_{1}^{i} S_{t}+a_{2}^{i} s_{t}^{i}+a_{3}^{i} s_{t}^{i} S_{t}} \\
& =\left(1+\frac{\left(a_{1}^{i}+a_{3}^{i} s_{t}^{i}\right) S_{t}\left(1-\lambda S_{t}\right) \alpha}{a_{0}^{i}+a_{1}^{i} S_{t}+a_{2}^{i} s_{t}^{i}+a_{3}^{i} s_{t}^{i} S_{t}}\right) \sigma_{c}+\frac{\left(a_{2}^{i}+a_{3}^{i} S_{t}\right) s_{t}^{i}\left(\mathbf{v}^{i}-\sum_{j=1}^{n} s_{t}^{j} \mathbf{v}^{j}\right)}{a_{0}^{i}+a_{1}^{i} S_{t}+a_{2}^{i} s_{t}^{i}+a_{3}^{i} s_{t}^{i} S_{t}}
\end{aligned}
$$

and again $\mu_{P, t}^{i}$ contains the "dt" terms. So expected returns are

$$
\mu_{R, t}^{i}=-\sigma_{P, t}^{i} \sigma_{m}^{\prime}
$$

Since the volatility vector of the pricing kernel is $\sigma_{m}=-\left(1+\alpha\left(1-S_{t} \lambda\right)\right) \sigma_{c}$, we obtain

$$
\begin{aligned}
\mu_{R, t}^{i}= & \left(1+\alpha\left(1-S_{t} \lambda\right)\right)\left(1+\frac{\left(a_{1}^{i}+a_{3}^{i} s_{t}^{i}\right) S_{t}\left(1-\lambda S_{t}\right) \alpha}{a_{0}^{i}+a_{1}^{i} S_{t}+a_{2}^{i} s_{t}^{i}+a_{3}^{i} s_{t}^{i} S_{t}}\right) \sigma_{c} \sigma_{c}^{\prime} \\
& +\frac{\left(1+\alpha\left(1-S_{t} \lambda\right)\right)\left(a_{2}^{i}+a_{3}^{i} S_{t}\right) s_{t}^{i}\left(\theta^{i}-\sum_{j=1}^{n} s_{t}^{j} \theta^{j}\right)}{a_{0}^{i}+a_{1}^{i} S_{t}+a_{2}^{i} s_{t}^{i}+a_{3}^{i} s_{t}^{i} S_{t}}
\end{aligned}
$$

Again, since we chose the $\theta^{i}$, s such that approximately $\sum_{j=1}^{n} s_{t}^{j} \theta^{j}=0$, to a first order we have $\mu_{R, t}^{i} \approx\left(1+\alpha\left(1-S_{t} \lambda\right)\right)\left\{\left(1+\frac{\left(a_{1}^{i}+a_{3}^{i} s_{t}^{i}\right) S_{t}\left(1-\lambda S_{t}\right) \alpha}{a_{0}^{i}+a_{1}^{i} S_{t}+a_{2}^{i} s_{t}^{i}+a_{3}^{i} s_{t}^{i} S_{t}}\right) \sigma_{c} \sigma_{c}^{\prime}+\frac{\left(a_{2}^{i}+a_{3}^{i} S_{t}\right) s_{t}^{i} \theta^{i}}{a_{0}^{i}+a_{1}^{i} S_{t}+a_{2}^{i} s_{t}^{i}+a_{3}^{i} s_{t}^{i} S_{t}}\right\}$ which implies that functions $\gamma_{1}\left(s_{t}^{i}, S_{t}\right)$ and $\gamma_{2}\left(s_{t}^{i}, S_{t}\right)$ in (33) are given by

$$
\begin{aligned}
\gamma_{1}\left(s_{t}^{i}, S_{t}\right) & =\frac{\left(a_{1}^{i}+a_{3}^{i} s_{t}^{i}\right)}{a_{0}^{i}+a_{1}^{i} S_{t}+a_{2}^{i} s_{t}^{i}+a_{3}^{i} s_{t}^{i} S_{t}} \\
\gamma_{2}\left(s_{t}^{i}, S_{t}\right) & =\frac{1}{\sigma_{c} \sigma_{c}^{\prime}} \frac{\left(a_{2}^{i}+a_{3}^{i} S_{t}\right)}{a_{0}^{i}+a_{1}^{i} S_{t}+a_{2}^{i} s_{t}^{i}+a_{3}^{i} s_{t}^{i} S_{t}}
\end{aligned}
$$


TABLE I

Railroads and Paper: 1927 - 2001

Panel A: Variance Decomposition

\begin{tabular}{ccccc} 
& Ret. Comp. & t-stat. & CF. Comp. & t-stat. \\
\hline Mkt Portfolio & -1.426 & -7.545 & 0.1466 & 0.9102 \\
Railroads & -0.3172 & -1.1726 & 0.3875 & 1.5291 \\
$\quad$ Paper & -1.3007 & -6.8039 & -0.2888 & -1.9991 \\
\hline
\end{tabular}

Panel B: Predictability Regressions

\begin{tabular}{cccc} 
& $\beta$ & t-stat. & $R^{2}$ \\
\hline Mkt Portfolio & -0.0196 & -2.4846 & $12.06 \%$ \\
Railroads & -0.0060 & -0.6934 & $0.78 \%$ \\
Paper & -0.0401 & -4.7262 & $21.06 \%$ \\
\hline
\end{tabular}


Table II

Description and Summary Statistics of Industries

\begin{tabular}{|c|c|c|c|c|c|}
\hline & $\begin{array}{l}\text { Industry } \\
\text { Description }\end{array}$ & $\mathrm{SIC}$ & $\begin{array}{l}\text { Avg. No. of } \\
\text { Stocks }\end{array}$ & $\begin{array}{l}\text { Min. No. of } \\
\text { Stocks }\end{array}$ & $\begin{array}{c}\text { Avg. Market } \\
\text { Cap. (\%) }\end{array}$ \\
\hline 1. & Mining & $10-14$ & 145.2 & 30 & 2.656 \\
\hline 2. & Food & 20 & 98 & 48 & 4.943 \\
\hline 3. & Apparel & $22-23$ & 74 & 18 & 0.609 \\
\hline 4. & Paper & 26 & 37.8 & 5 & 1.904 \\
\hline 5. & Chemical & 28 & 150.5 & 25 & 10.394 \\
\hline 6. & Petroleum & 29 & 35.4 & 23 & 10.610 \\
\hline 7. & Construction & 32 & 36.2 & 5 & 1.273 \\
\hline 8. & Prim. Metals & 33 & 75.7 & 44 & 4.269 \\
\hline 9. & Fab. Metals & 34 & 73.6 & 9 & 1.415 \\
\hline 10. & Machinery & 35 & 185.7 & 25 & 5.760 \\
\hline 11. & Electric Eq. & 36 & 198.9 & 14 & 6.064 \\
\hline 12. & Transport Eq. & 37 & 91.4 & 46 & 7.646 \\
\hline 13. & Manufacturing & $38-39$ & 153.6 & 10 & 2.902 \\
\hline 14. & Railroads & 40 & 34 & 8 & 3.049 \\
\hline 15. & Other Transport. & $41-47$ & 61.4 & 15 & 0.875 \\
\hline 16. & Utilities & 49 & 127.7 & 21 & 7.856 \\
\hline 17. & Dept. Stores & 53 & 42.4 & 20 & 3.743 \\
\hline 18. & Retail & $50-52 \quad 54-59$ & 254.2 & 22 & 2.313 \\
\hline 19. & Financial & $60-69$ & 441.6 & 15 & 6.927 \\
\hline 20. & Other & & 619.2 & 57 & 14.788 \\
\hline
\end{tabular}


TABLE III

Model Parameters

\begin{tabular}{|c|c|c|c|c|c|c|c|}
\hline \multicolumn{8}{|c|}{ Panel A: Calibrated Parameters } \\
\hline & $\mathrm{k}$ & $\bar{Y}$ & $\lambda$ & $\alpha$ & $\phi$ & $\mu_{C}$ & $\sigma_{C}$ \\
\hline & 0.0506 & 29.6 & 9.7922 & 53.0621 & 0.0527 & 0.0173 & 0.0177 \\
\hline \multicolumn{8}{|c|}{ Panel B: Parameters of Share Process } \\
\hline Ptfl No. & Name & $\bar{s}^{i}(\%)$ & $\theta_{i}(\%)$ & Ptfl No. & Name & $\bar{s}^{i}(\%)$ & $\theta_{i}(\%)$ \\
\hline 1 & Mining & 0.01528 & -0.02836 & 11 & Electrical E. & 0.41537 & 0.02086 \\
\hline 2 & Food & 0.24393 & -0.01399 & 12 & Transport Eq. & 0.68679 & 0.02456 \\
\hline 3 & Apparel & 0.03653 & -0.04168 & 13 & Manufacturing & 0.05596 & -0.0136 \\
\hline 4 & Paper & 0.12313 & -0.02534 & 14 & Railroads & 0.00166 & 0.0126 \\
\hline 5 & Chemical & 0.57202 & -0.02536 & 15 & Other Transp. & 0.04848 & 0.02504 \\
\hline 6 & Petroleum & 0.46272 & 0.00557 & 16 & Utilities & 0.55889 & 0.00415 \\
\hline 7 & Construction & 0.17422 & -0.04336 & 17 & Dept. Stores & 0.09263 & -0.03049 \\
\hline 8 & Prim. Metals & 0.15567 & 0.00689 & 18 & Retail & 0.07681 & -0.02541 \\
\hline 9 & Fab. Metals & 0.09226 & -0.01187 & 19 & Financial & 0.68679 & 0.03709 \\
\hline 10 & Machinery & 0.37739 & 0.00626 & 20 & Other & 0.53999 & -0.02094 \\
\hline Mean & $\begin{array}{l}\text { Reversion } \\
\qquad \sum \bar{s}^{i}(\%)\end{array}$ & $\kappa(\%)$ & $\begin{array}{l}1.04336 \\
5.41651 \\
\end{array}$ & & & & \\
\hline
\end{tabular}

Panel A: Preference parameters calibrated to match the sample average of market excess return, the sample average risk free rate, the volatility of the risk free rate, the average price consumption ratio of the market portfolio, and the average Sharpe ratio. $\mu_{C}$ and $\sigma_{C}$ are obtained from NIPA tables. Panel B: $\left\{\kappa, \bar{s}^{1}, . ., \bar{s}^{n}\right\}$ are estimated using a two stage GMM procedure with NW corrected variance covariance matrix applied to the Euler discretization of the process $(4) .\left(\theta^{1}, \cdots, \theta^{n}\right)$ are computed from the vectors $\mathbf{v}^{i}$, from the variance-covariance matrix of the process for the $\log s_{t}^{i}$, where we have assumed that the term $\sum_{j=1}^{n} s_{t}^{j} \mathbf{v}^{j}$ that appears in the volatility function in (4) is approximately constant. 
TABLE IV

Averages and Standard Deviations

Panel A: Aggregate Variables

\begin{tabular}{lcccccc} 
& $\mu_{R}^{*}$ & $\sigma_{R}$ & $r_{f}^{*}$ & $\sigma_{r}^{*}$ & Ave $\mathrm{PD}^{*}$ & $\mathrm{SR}^{*}$ \\
\hline Model & 6.654 & 20.272 & 0.716 & 1.265 & 31.229 & 0.334 \\
Data & 7.345 & 22.034 & 1.015 & 2.022 & 29.612 & 0.333 \\
\hline
\end{tabular}

Panel B: Industries

\begin{tabular}{cccccccccc} 
& & \multicolumn{2}{c}{ Avg. Return } & \multicolumn{2}{c}{ Avg. P/C } & \multicolumn{2}{c}{ Avg P/D } & \multicolumn{2}{c}{ Sharpe Ratio } \\
& & Data & Model & Data & Model & Data & Model & Data & Model \\
\hline 1 & Mining & 6.586 & 6.656 & 0.020 & 0.004 & 36.586 & 49.593 & 0.287 & 0.268 \\
2 & Food & 8.226 & 6.449 & 0.036 & 0.076 & 30.122 & 34.100 & 0.417 & 0.319 \\
3 & Apparel & 6.365 & 6.152 & 0.005 & 0.013 & 35.559 & 45.126 & 0.201 & 0.282 \\
4 & Paper & 8.875 & 6.377 & 0.016 & 0.040 & 31.063 & 38.951 & 0.322 & 0.297 \\
5 & Chemical & 9.241 & 6.110 & 0.082 & 0.184 & 34.036 & 34.035 & 0.397 & 0.329 \\
6 & Petroleum & 8.984 & 6.876 & 0.074 & 0.136 & 23.784 & 31.940 & 0.436 & 0.326 \\
7 & Construction & 7.338 & 6.878 & 0.009 & 0.122 & 32.0247 & 82.902 & 0.242 & 0.244 \\
8 & Prim. Metals & 6.862 & 7.395 & 0.027 & 0.054 & 31.628 & 46.512 & 0.217 & 0.275 \\
9 & Fab. Metals & 7.063 & 6.533 & 0.010 & 0.028 & 32.662 & 34.108 & 0.287 & 0.319 \\
10 & Machinery & 9.137 & 7.016 & 0.050 & 0.113 & 49.197 & 34.914 & 0.326 & 0.308 \\
11 & Electrical E. & 9.750 & 7.341 & 0.055 & 0.133 & 40.953 & 34.005 & 0.322 & 0.308 \\
12 & Transport Eq. & 9.409 & 7.499 & 0.056 & 0.201 & 22.191 & 36.867 & 0.306 & 0.297 \\
13 & Manufacturing & 8.315 & 6.435 & 0.026 & 0.017 & 48.659 & 33.487 & 0.350 & 0.324 \\
14 & Railroads & 7.846 & 7.160 & 0.017 & 0.001 & 27.509 & 35.017 & 0.258 & 0.306 \\
15 & Other Transp. & 6.170 & 7.520 & 0.007 & 0.013 & 52.413 & 36.277 & 0.224 & 0.298 \\
16 & Utilities & 6.309 & 6.757 & 0.059 & 0.167 & 20.094 & 30.245 & 0.276 & 0.339 \\
17 & Dept. Stores & 8.679 & 6.077 & 0.027 & 0.028 & 39.734 & 36.694 & 0.333 & 0.314 \\
18 & Retail & 8.211 & 6.509 & 0.021 & 0.023 & 45.045 & 42.251 & 0.351 & 0.285 \\
19 & Financial & 8.464 & 7.589 & 0.070 & 0.185 & 28.976 & 31.489 & 0.302 & 0.317 \\
20 & Other & 6.525 & 6.218 & 0.125 & 0.169 & 34.311 & 33.519 & 0.332 & 0.330 \\
\hline
\end{tabular}

Panel A: * Statistics that the model was chosen to replicate. Model generated excess market returns, market returns volatility, average risk free rate and its volatility, average market price dividend ration, market Sharpe ratio versus the model generated counterparts. Panel B. Sample average returns, average price consumption ratios, average price dividend ratios, and average Sharpe ratios versus the simulated averages for the set of 20 industry portfolios. The model is simulated at the quarterly frequency. All return are annualized. 
TABLE V

Predictability

Panel A: Market Portfolio

\begin{tabular}{lcccccccc} 
& \multicolumn{2}{c}{1 year } & \multicolumn{2}{c}{2 years } & \multicolumn{2}{c}{3 years } & \multicolumn{2}{c}{5 years } \\
& $\beta$ & $R^{2}$ & $\beta$ & $R^{2}$ & $\beta$ & $R^{2}$ & $\beta$ & $R^{2}$ \\
\hline Model & -0.3024 & 0.1085 & -0.4976 & 0.1773 & -0.6216 & 0.2194 & -0.7720 & 0.2670 \\
Data & -0.1084 & 0.0513 & -0.1808 & 0.0656 & -0.2460 & 0.0779 & -0.3920 & 0.1206 \\
Data $(1947-1994)$ & -0.2692 & 0.1755 & -0.4768 & 0.2937 & -0.6696 & 0.4209 & -1.0480 & 0.5614 \\
\hline
\end{tabular}

Panel B: Predictability Across Industries

\begin{tabular}{|c|c|c|c|c|c|c|c|c|c|}
\hline & & \multicolumn{4}{|c|}{2 year predictability } & \multicolumn{4}{|c|}{5 year predictability } \\
\hline & & & & -1 & - & & - & -1 & - \\
\hline & & Model & Data & Model & Data & Model & Data & Model & Data \\
\hline 1 & Mining & -0.1240 & -0.1512 & 0.0311 & 0.0476 & -0.1300 & -0.3420 & 0.0506 & 0.1043 \\
\hline 2 & Food & -0.3616 & -0.1064 & 0.1020 & 0.0284 & -0.5380 & -0.2020 & 0.1414 & 0.0415 \\
\hline 3 & Apparel & -0.1888 & -0.2024 & 0.0467 & 0.0826 & -0.2520 & -0.4700 & 0.0713 & 0.1682 \\
\hline 4 & Paper & -0.2664 & -0.2760 & 0.0678 & 0.0629 & -0.3840 & -0.8020 & 0.0961 & 0.2106 \\
\hline 5 & Chemical & -0.4000 & -0.2744 & 0.1294 & 0.0839 & -0.6080 & -0.5480 & 0.1825 & 0.1391 \\
\hline 6 & Petroleum & -0.3864 & -0.4488 & 0.1060 & 0.1441 & -0.5820 & -0.8960 & 0.1496 & 0.2687 \\
\hline 7 & Construction & 0.0016 & -0.1248 & 0.0174 & 0.0208 & 0.0640 & -0.3480 & 0.0452 & 0.0710 \\
\hline 8 & Prim. Metals & -0.1200 & -0.0088 & 0.0308 & 0.0001 & -0.1360 & -0.1400 & 0.0543 & 0.0142 \\
\hline 9 & Fab. Metals & -0.3640 & -0.1736 & 0.1020 & 0.0511 & -0.5380 & -0.3360 & 0.1416 & 0.0900 \\
\hline 10 & Machinery & -0.3064 & -0.0552 & 0.0757 & 0.0063 & -0.4420 & -0.2560 & 0.1062 & 0.0484 \\
\hline 11 & Electrical E. & -0.3000 & -0.1616 & 0.0697 & 0.0286 & -0.4320 & -0.4400 & 0.0976 & 0.0790 \\
\hline 12 & Transport Eq. & -0.2368 & -0.1424 & 0.0526 & 0.0178 & -0.3180 & -0.3100 & 0.0770 & 0.0395 \\
\hline 13 & Manufacturing & -0.3832 & -0.1072 & 0.1120 & 0.0289 & -0.5780 & -0.3280 & 0.1580 & 0.1062 \\
\hline 14 & Railroads & -0.2952 & -0.1048 & 0.0699 & 0.0150 & -0.4260 & -0.1200 & 0.0997 & 0.0078 \\
\hline 15 & Other Transp. & -0.2472 & -0.0584 & 0.0559 & 0.0099 & -0.3480 & -0.1700 & 0.0821 & 0.0291 \\
\hline 16 & Utilities & -0.4600 & -0.3392 & 0.1471 & 0.1146 & -0.7060 & -0.8380 & 0.2126 & 0.3064 \\
\hline 17 & Dept. Stores & -0.3352 & -0.1720 & 0.0981 & 0.0616 & -0.5000 & -0.5420 & 0.1382 & 0.2155 \\
\hline 18 & Retail & -0.2000 & -0.1224 & 0.0484 & 0.0583 & -0.2660 & -0.2540 & 0.0710 & 0.1081 \\
\hline 19 & Financial & -0.3536 & -0.3536 & 0.0833 & 0.1206 & -0.5200 & -0.8760 & 0.1186 & 0.2820 \\
\hline 20 & Other & -0.4072 & -0.0912 & 0.1317 & 0.0253 & -0.6220 & -0.1500 & 0.1867 & 0.0207 \\
\hline
\end{tabular}

Panel A. Long horizon return regressions. The first line corresponds to the long average statistics of the long horizon regressions in artificial data. The second line corresponds to its sample counterpart for the period 1927-2001. The third line corresponds to the sample period, 1947-1994. Panel B. Long horizon regressions, 2 and 5 year, across industries both in artificial data (Model) and historical data. 
TABLE VI Variance Decomposition

Panel A: Market Portfolio

\begin{tabular}{lcc} 
& CF Component & Return Component \\
\hline Model Dividend & 0.12442 & -0.79127 \\
Model Consumption & -0.04039 & -0.95855 \\
$\quad$ Data & 0.14655 & -1.14686 \\
\hline
\end{tabular}

Panel B: Industries

\begin{tabular}{clcccc} 
& & Cash Flow Component & \multicolumn{2}{c}{ Return Component } \\
& & Model & Data & Model & Data \\
\hline 1 & Mining & 0.89906 & 0.4793 & -0.02241 & -0.48552 \\
2 & Food & 0.31642 & 0.41623 & -0.5988 & -0.59811 \\
3 & Apparel & 0.73706 & 0.1969 & -0.18315 & -0.65425 \\
4 & Paper & 0.54955 & -0.28881 & -0.36862 & -1.12882 \\
5 & Chemical & 0.20923 & 0.10608 & -0.70983 & -1.13722 \\
6 & Petroleum & 0.25367 & 0.41863 & -0.65961 & -1.12375 \\
7 & Construction & 1.18856 & 0.09522 & -0.21573 & -1.00528 \\
8 & Prim. Metals & 0.94517 & 0.78275 & -0.00163 & -0.2155 \\
9 & Fab. Metals & 0.32465 & 0.14027 & -0.59182 & -0.69194 \\
10 & Machinery & 0.46244 & 0.24976 & -0.45759 & -0.92006 \\
11 & Electrical E. & 0.4745 & 0.18175 & -0.44513 & -1.08215 \\
12 & Transport Eq. & 0.65107 & 0.32261 & -0.2774 & -0.70583 \\
13 & Manufacturing & 0.25306 & 0.31889 & -0.6605 & -0.90606 \\
14 & Railroads & 0.50692 & 0.38748 & -0.41424 & -0.24663 \\
15 & Other Transp. & 0.60819 & -0.03385 & -0.31892 & -0.64547 \\
16 & Utilities & 0.05997 & -0.11848 & -0.85534 & -1.10745 \\
17 & Dept. Stores & 0.37063 & 0.18955 & -0.54601 & -0.99149 \\
18 & Retail & 0.73414 & 0.22496 & -0.18888 & -0.3239 \\
19 & Financial & 0.34233 & 0.64092 & -0.57919 & -1.10387 \\
20 & Other & 0.18109 & 0.37208 & -0.73773 & -0.51911 \\
\hline & & & & &
\end{tabular}

Panel A: Variance decomposition of the log price-dividend ratio (line 1) and of the log price consumption ratio (line 2) in artificial data for the market portfolio. Line 3 is the variance decomposition of the $\log$ price dividend ratio in historical data. Panel B: Variance decomposition of the price-dividend ratio across industries both in artificial data (Model) and historical data. 
TABLE VII

Correlation Market P/D Ratio with Dispersion of Industry P/D Ratios

\begin{tabular}{ccccc} 
Panel A: Standard Deviation of Industry P/D's \\
& \multicolumn{2}{c}{$\log (\mathrm{P} / \mathrm{D})$} & \multicolumn{2}{c}{ P/D } \\
& Level & First Diff. & Level & First Diff. \\
\hline Model & 0.2279 & 0.2761 & 0.4435 & 0.5232 \\
Data & 0.7517 & 0.4051 & 0.8941 & 0.7539 \\
\hline \multicolumn{4}{c}{} \\
Panel B: Absolute Deviation of Industry P/D's \\
\multicolumn{4}{c}{$\log (\mathrm{P} / \mathrm{D})$} & \multicolumn{2}{c}{ P/D } \\
Level & First Diff. & Level & First Diff. \\
\hline Datel & 0.2859 & 0.3469 & 0.5436 & 0.6388 \\
\hline & 0.8022 & 0.3844 & 0.9420 & 0.7989 \\
\hline
\end{tabular}


Figure 1: Market log PD and Cross-Sectional Dispersion of Industries log PDs
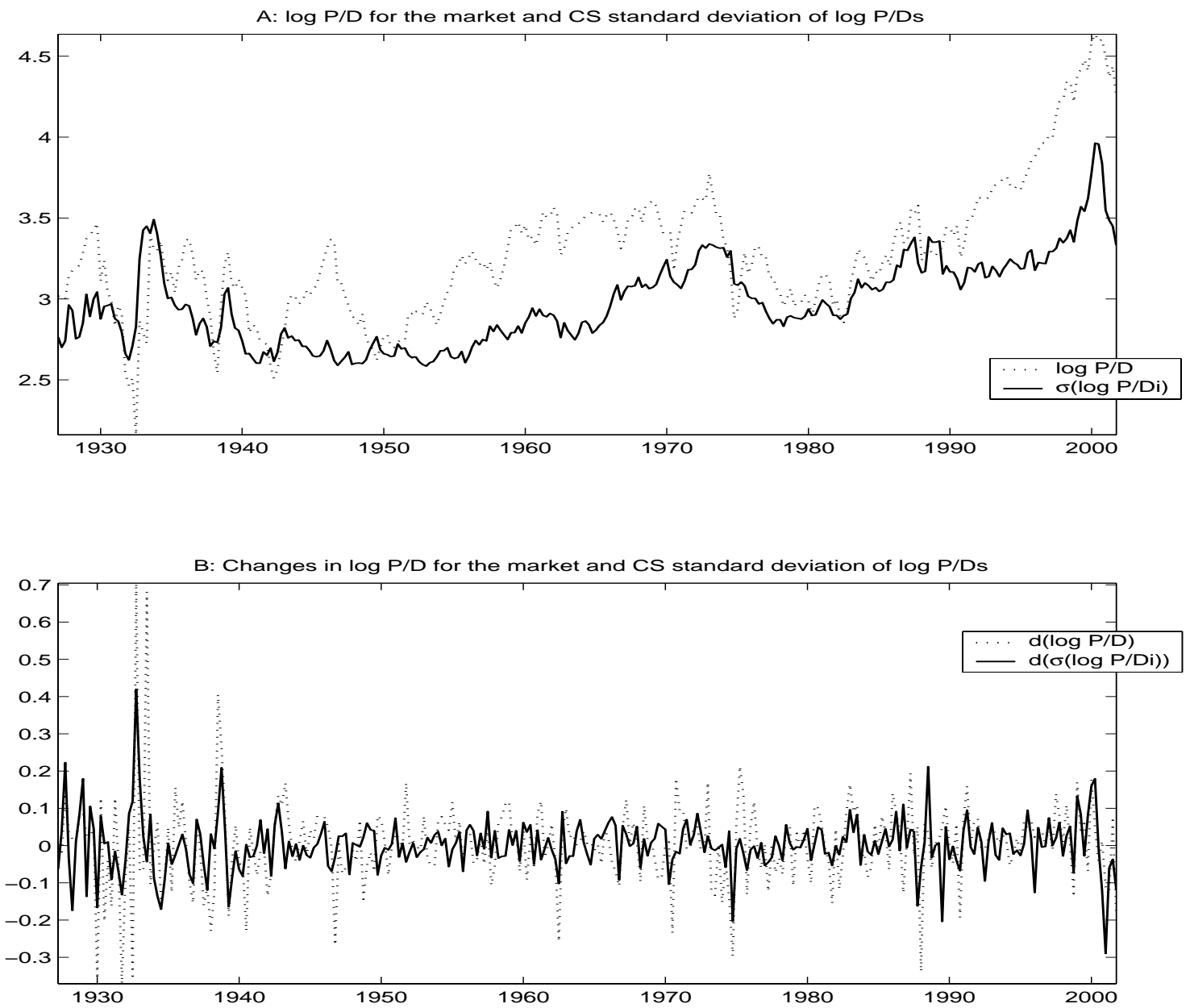

Panel A: Log price dividend ratio of the market portfolio versus the (rescaled) cross sectional standard deviation of the log price dividend ratios of the 20 industry portfolios for the sample period 1927-2001. Panel B: Plots of the same time series as in Panel A but in first differences. 
Figure 2: Railroads and Paper

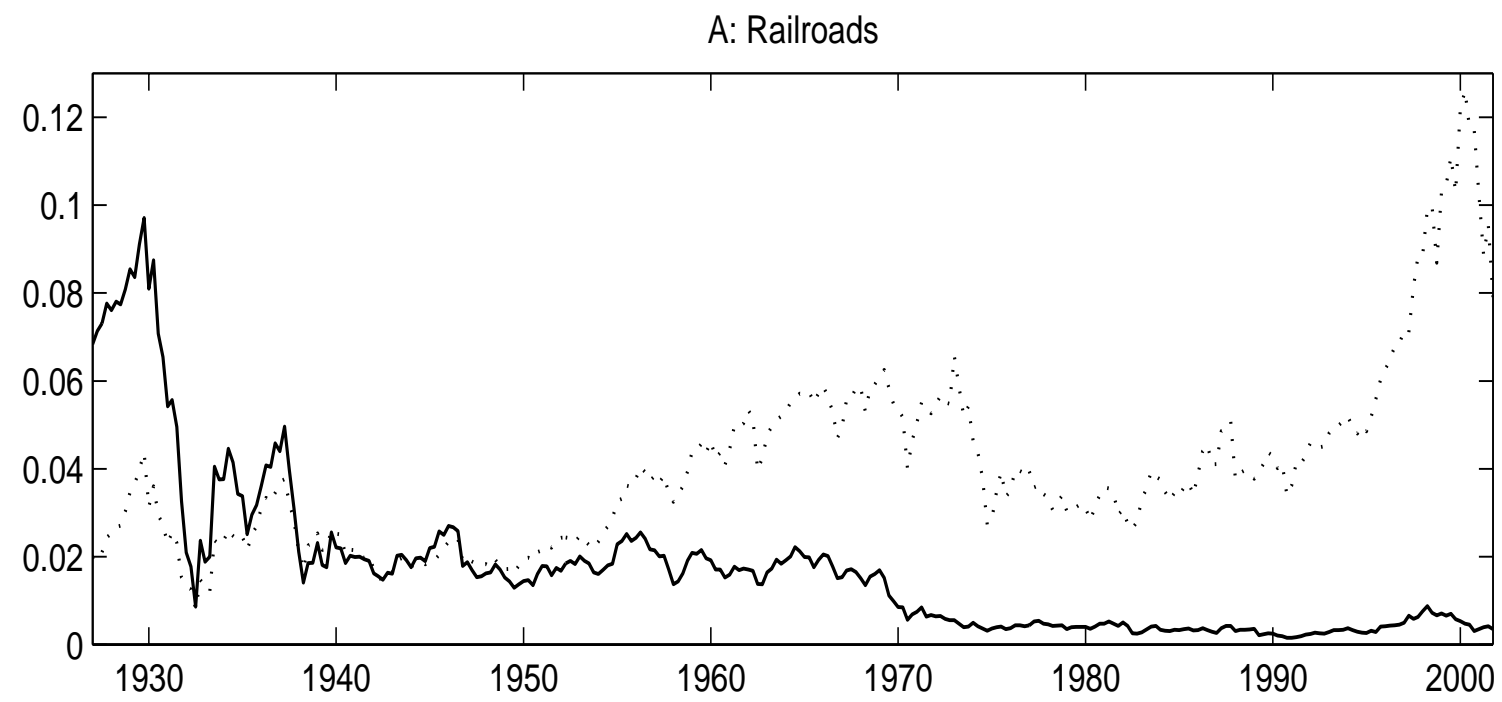

B: Paper

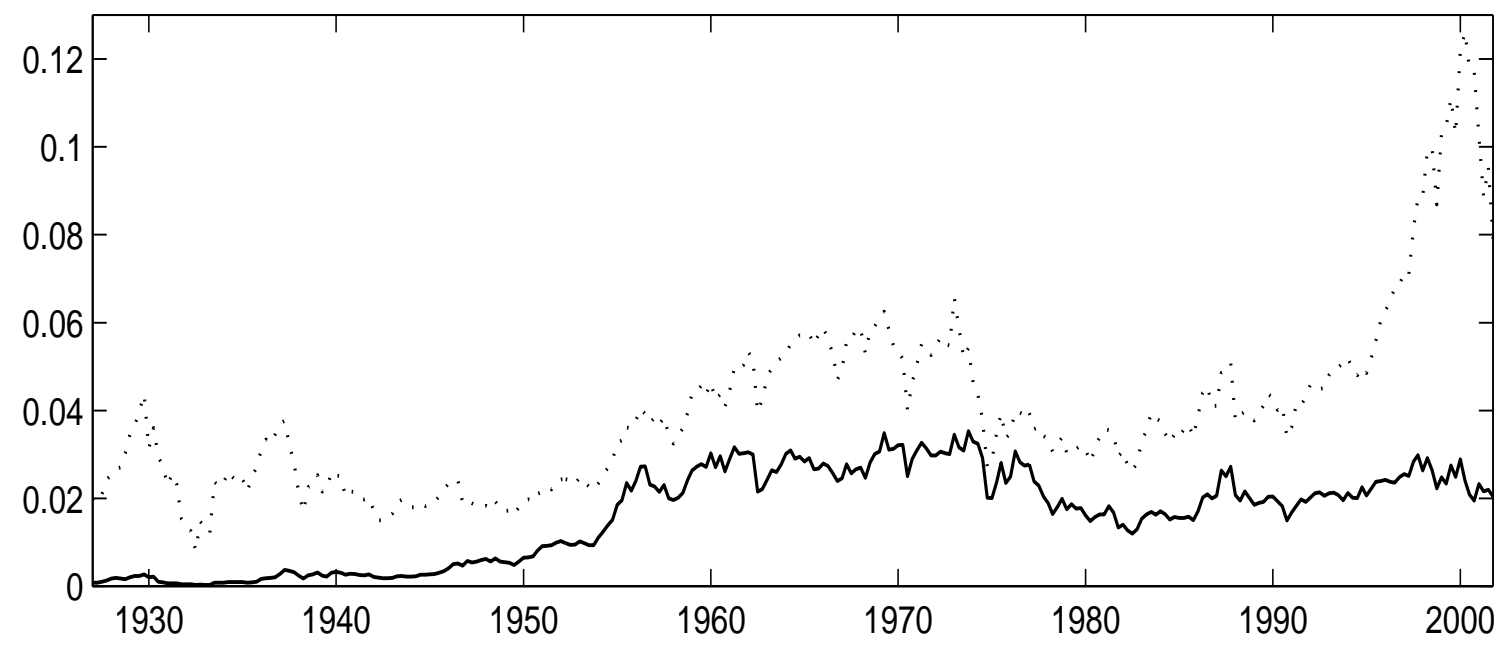

Price consumption ratio of Railroads (Panel A) and Paper (Panel B) for the sample period of 1927-2001. The dotted line corresponds to a rescaled price consumption ratio of the market portfolio. 
Figure 3: Aggregate Quantities
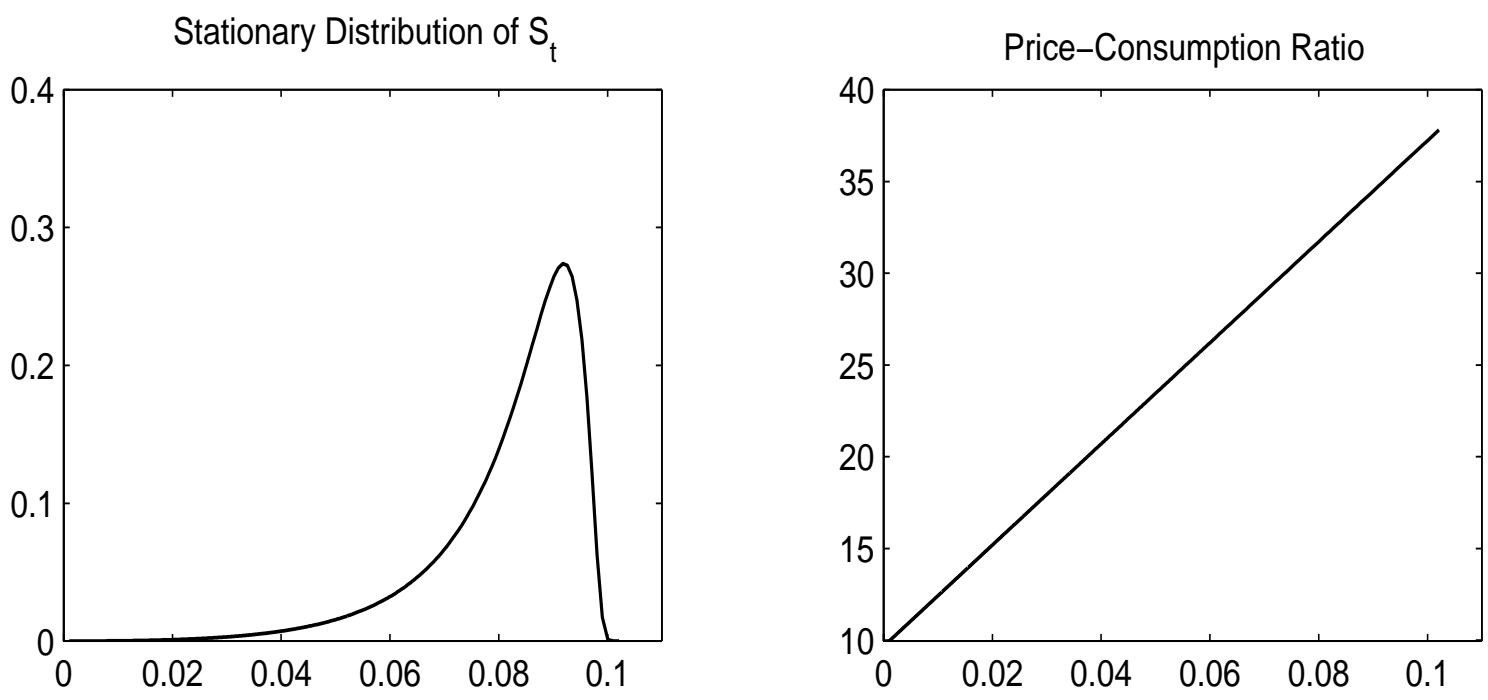

Expected Returns, Volatility and Interest Rate

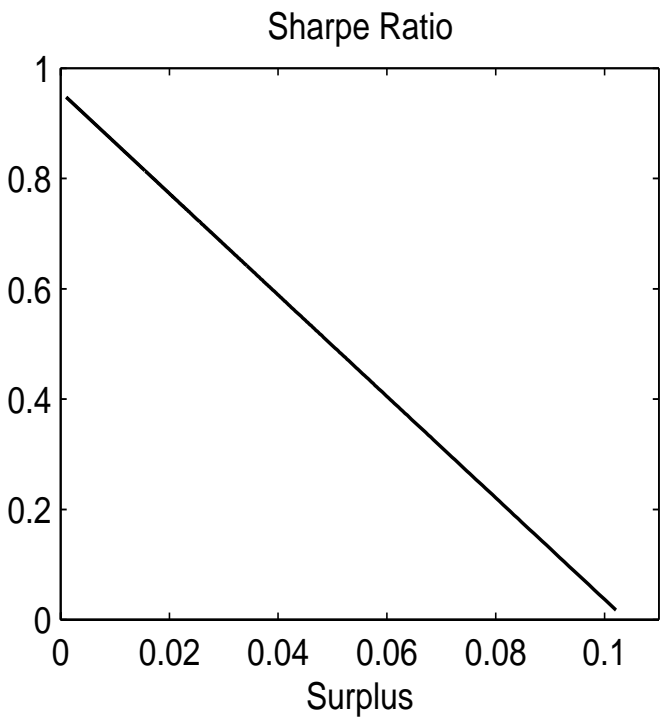

Panel A: Stationary density function of $S_{t}$. Panel B. Price consumption ratio of the total wealth portfolio as a function of $S_{t}$. Panel C: Expected returns and volatility of returns of the total wealth portfolio as a function of $S_{t}$ and the risk free rate. Panel D: Sharpe ratio of the total wealth portfolio as a function of $S_{t}$. The parameters used are those of Table III. 
Figure 4: Model Performance: Unconditional Moments
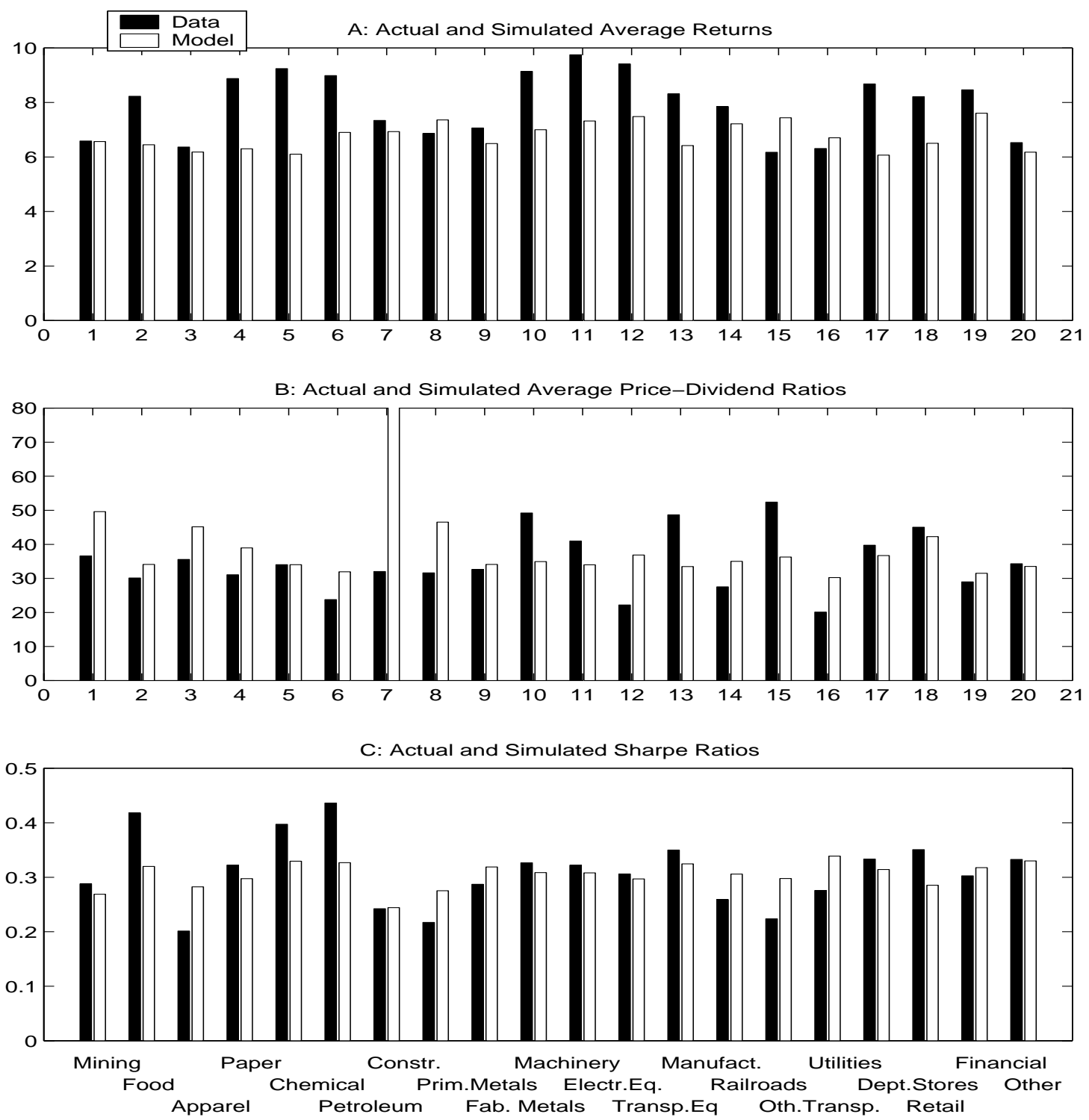

Panel A: Sample average returns versus average returns in simulated data across industries. Panel B: Sample average price dividend ratios versus average price dividend ratios in simulated data across industries. Panel C: Sample average Sharpe ratios versus average Sharpe ratios in simulated data across industries. Numbers are reported in Table IV. 
Figure 5: Model Performance: Predictability
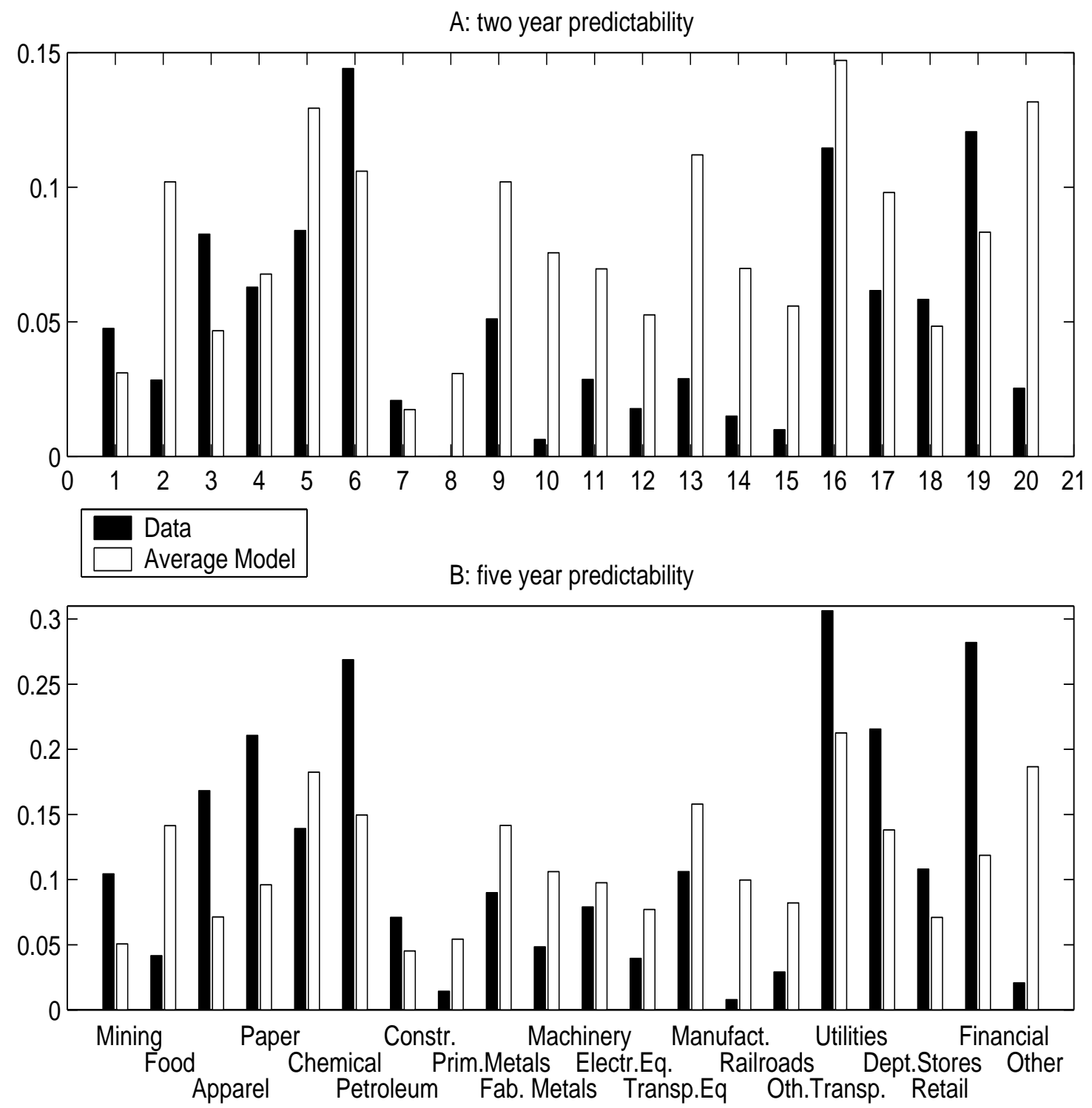

Panel A: $\mathrm{R}^{2}$ corresponding to the two year predictability regression across industries both in historical and simulated data. Panel $\mathrm{B}: \mathrm{R}^{2}$ corresponding to the five year predictability regression across industries both in historical and simulated data. Numbers are reported in Table V. 
Figure 6: Model Performance: Variance Decomposition
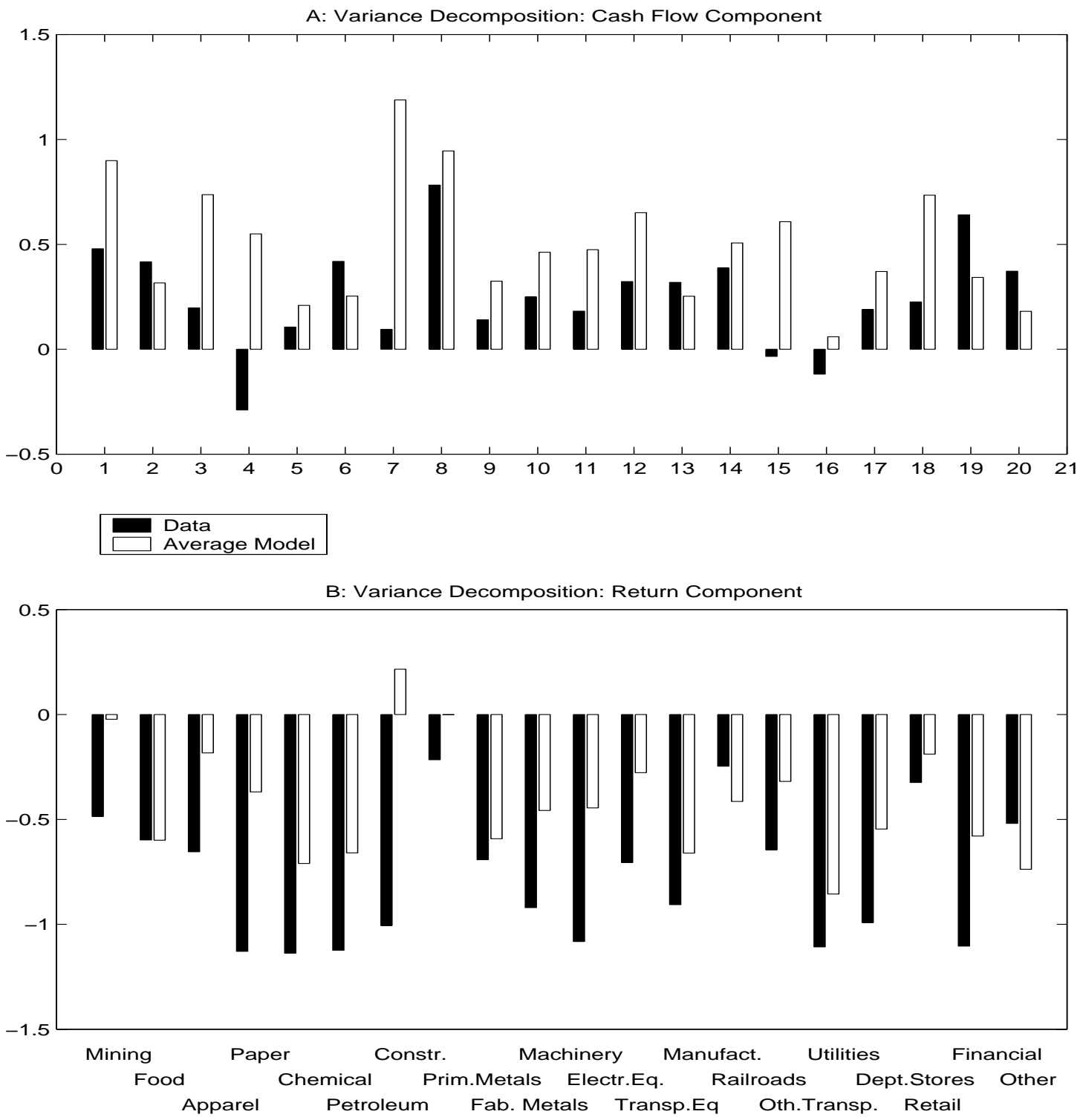

Panel A: Cash flow component of the variance decomposition of the log pricedividend ratio across industries both in historical and simulated data. Panel B: Return component of the variance decomposition of the log price-dividend ratio across industries both in historical and simulated data. Numbers are reported in Table VI. 
Figure 7: Time Series: Surplus, Habit and Market log P/D
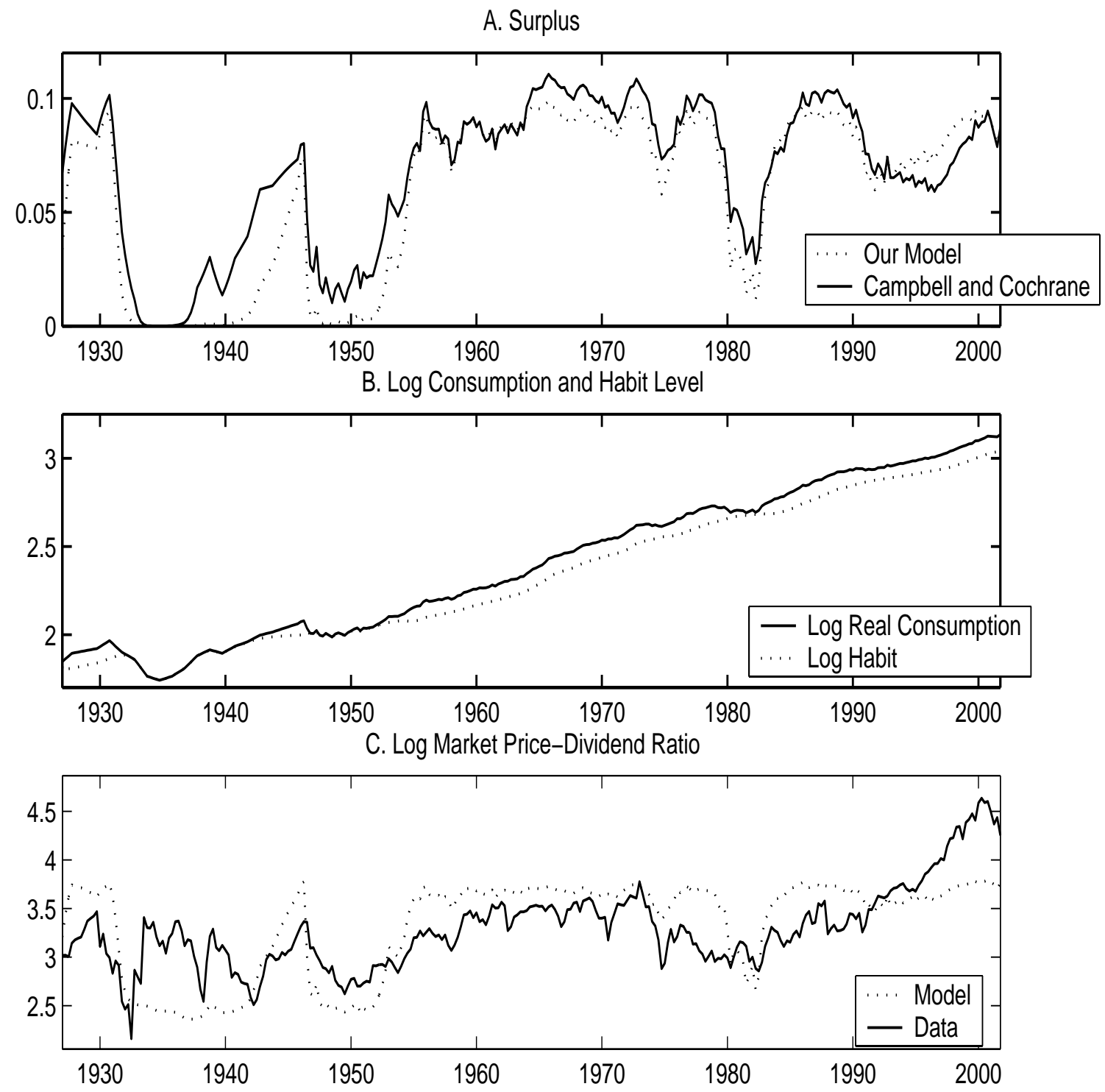

Panel A: Historical surplus consumption ratio given realized consumption shocks both in the model and the one obtained by Campbell and Cochrane (1999). Panel B: Nondurable and services consumption per capita and habit level implied by the model. Panel C: Log market price dividend ratio both in historical data (continuous line) and model implied when fed the observed consumption and dividend data (dotted line). 
Figure 8: Time Series P/C ratios: Industry 1 - 10
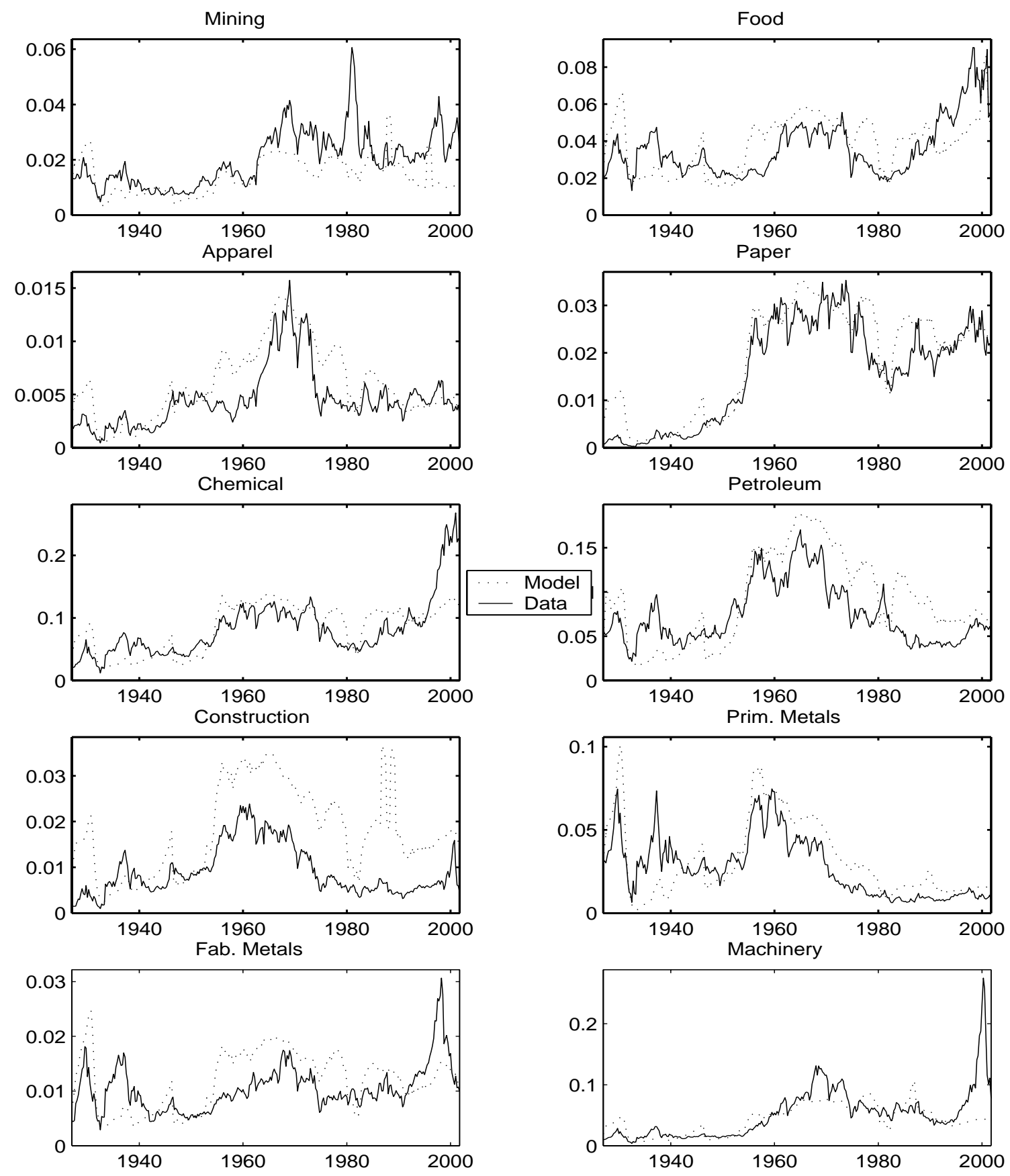

See Notes after Figure 9. 
Figure 9: Time Series P/C ratios: Industry $11-20$
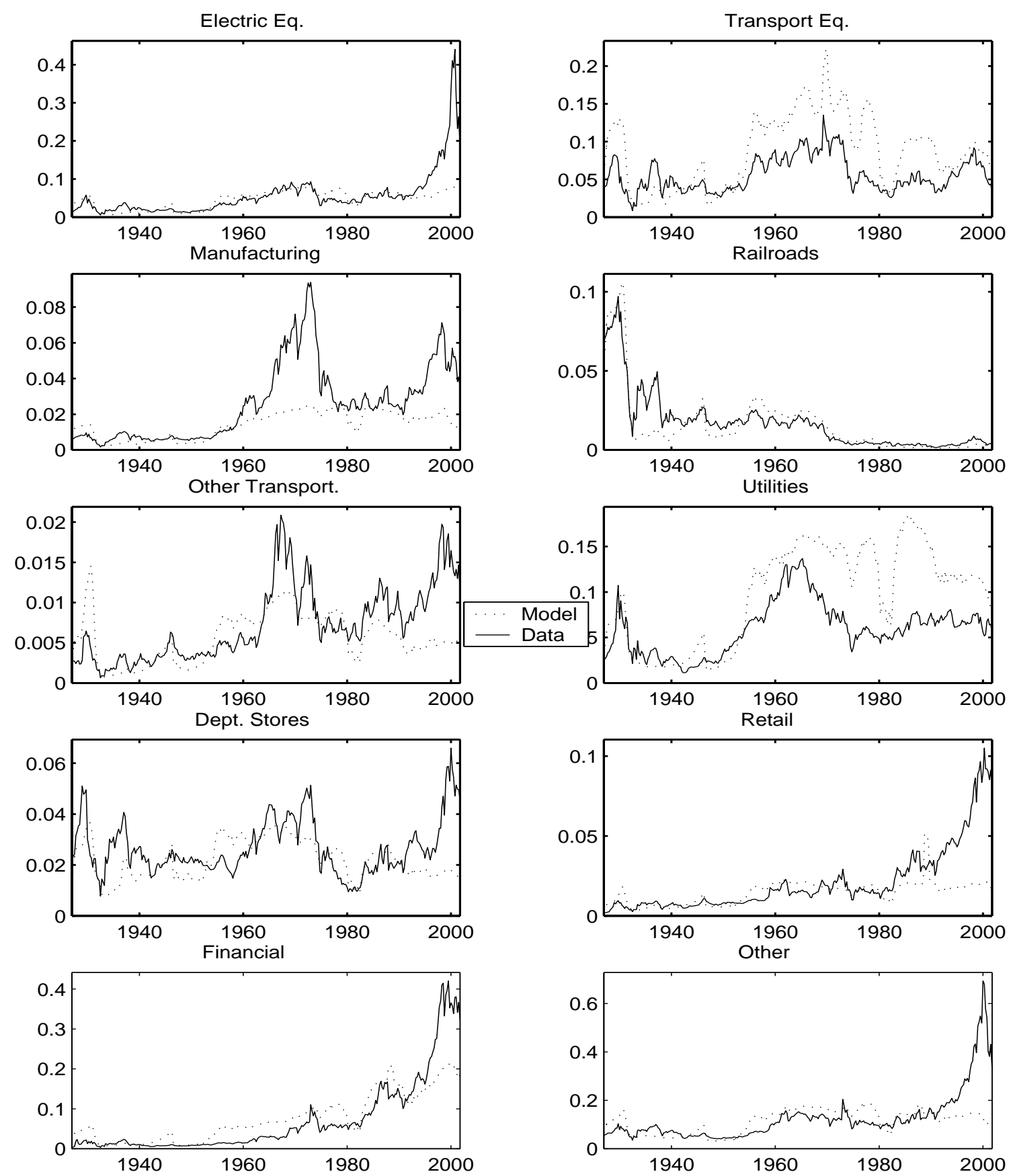

Price consumption ratio both in historical data (continuous line) versus model implied when fed the observed consumption and dividend data (dotted line) across the 20 industries. 
Figure 10: Shares and P/Cs: Railroads and Paper
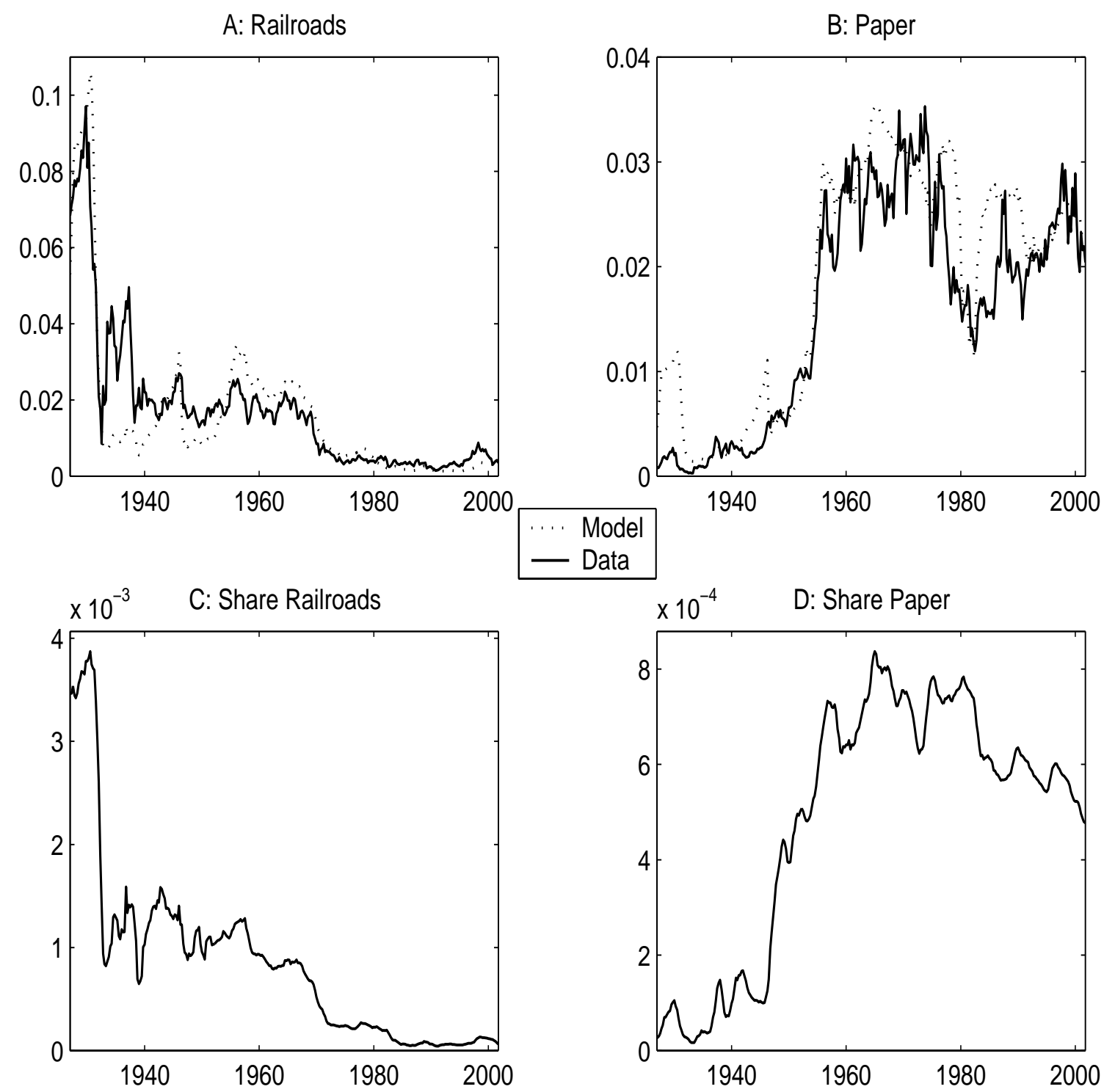

Panels A and B: Historical Price consumption ratio of Paper and Railroads (continuous line) versus model implied when fed the observed consumption and dividend data (dotted line). Panels $\mathrm{C}$ and $\mathrm{D}$ : Realized dividend consumption shares for Railroads and Paper. 
Figure 11: Market Behavior and the Cross-Sectional Dispersion of $\mathrm{P} / \mathrm{Cs}$ and $\mathrm{P} / \mathrm{Ds}$

A. Cross-Sectional Standard Deviation of Price-Consumption Ratios

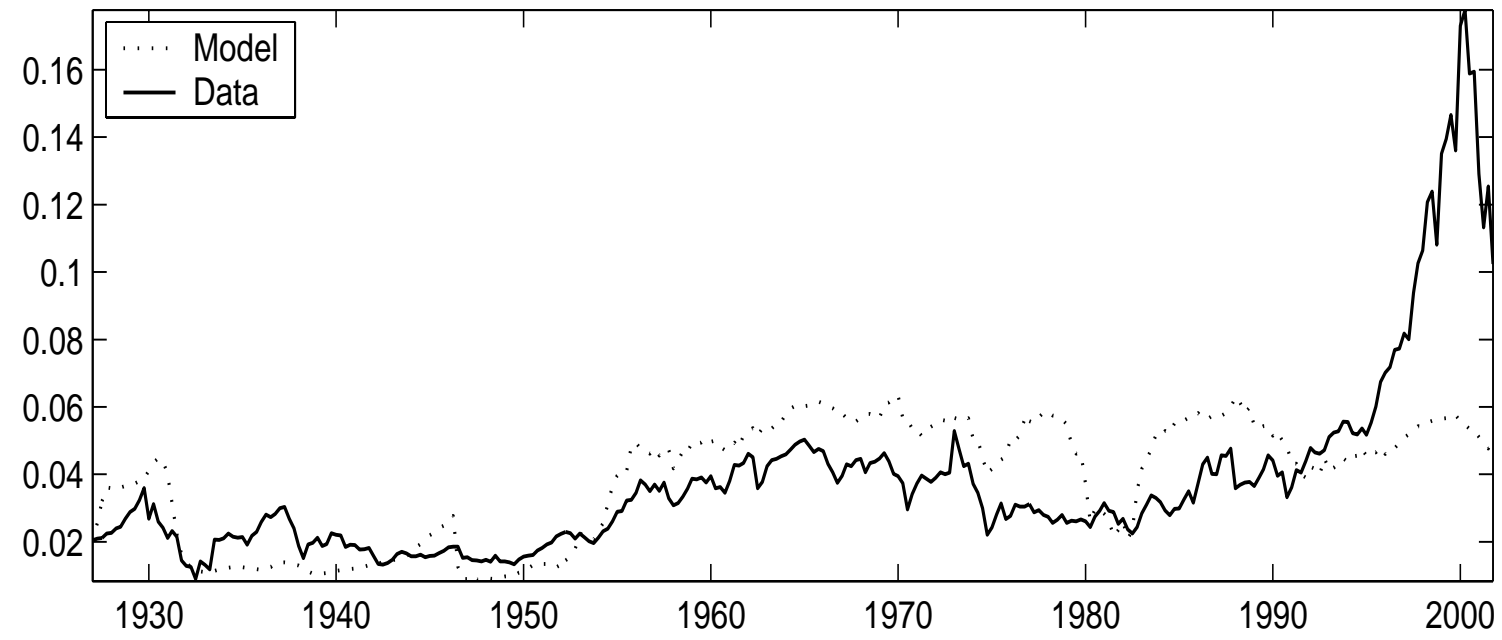

B. Cross-Sectional Standard Deviation of Price-Dividend Ratios

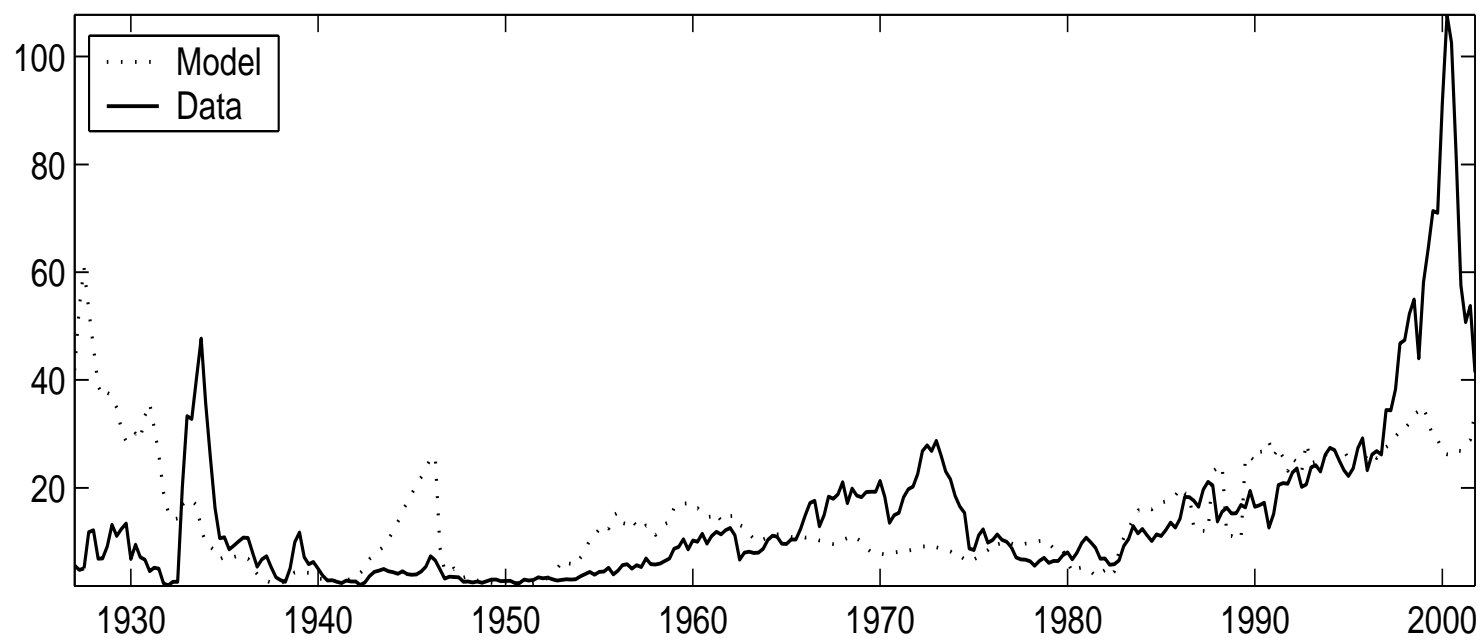

Panel A: Historical cross sectional standard deviation of price consumption ratios (continuous line) versus model implied when fed the observed consumption and dividend data (dotted line). Panel B: Historical cross sectional standard deviation of price dividend ratios (continuous line) versus model implied when fed the observed consumption and dividend data (dotted line). 
Figure 12: Market Implied Long-Term Shares $\bar{s}^{i}$

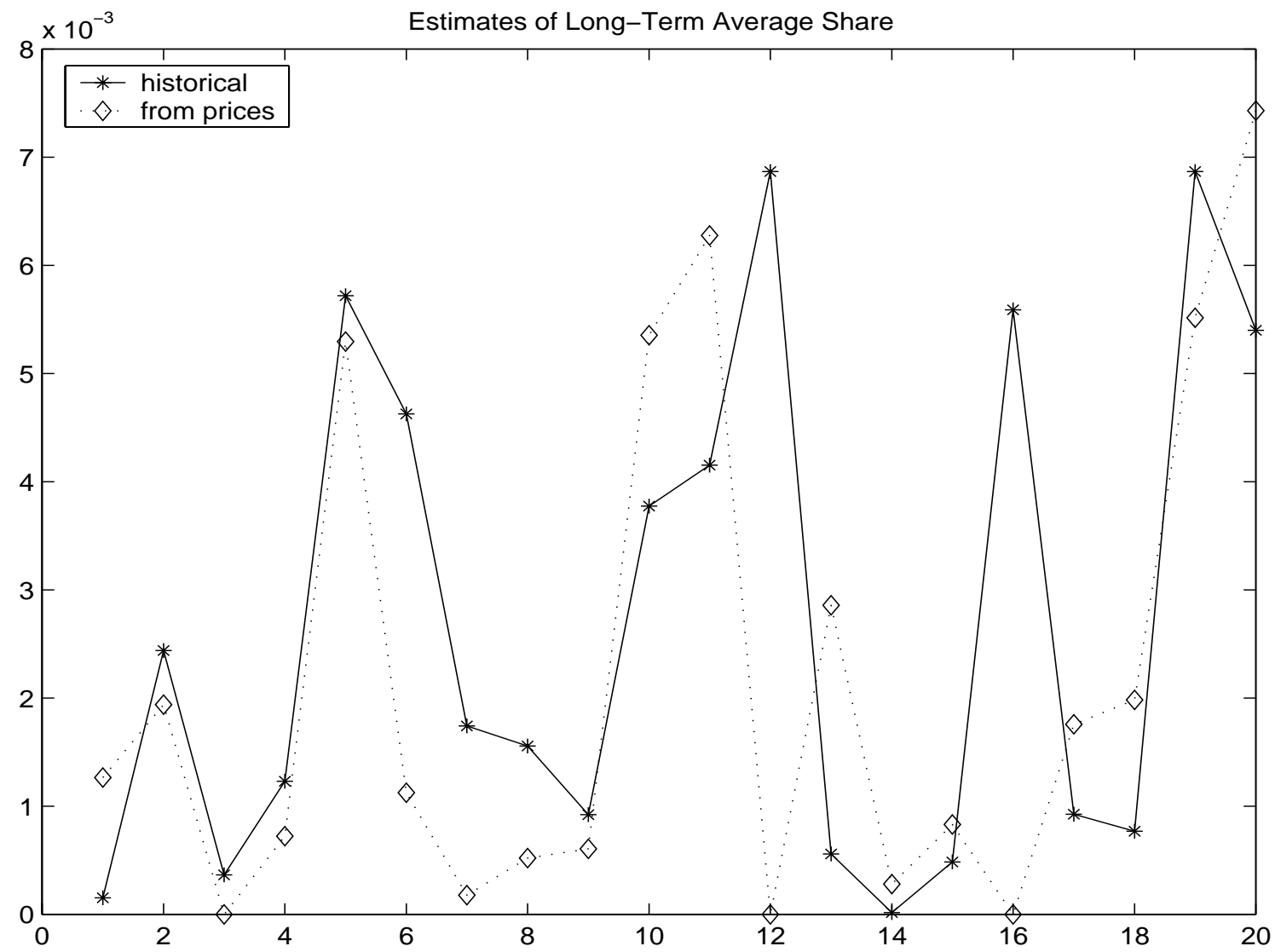

Sample average shares across industries versus the ones estimated to match the average price consumption ratio of the corresponding industry. 